\title{
Search for Top Squark and Higgsino Production Using Diphoton Higgs Boson Decays
}

\author{
S. Chatrchyan et al. ${ }^{*}$ \\ (CMS Collaboration) \\ (Received 11 December 2013; published 25 April 2014)
}

\begin{abstract}
Results are presented of a search for a "natural" supersymmetry scenario with gauge mediated symmetry breaking. It is assumed that only the supersymmetric partners of the top quark (the top squark) and the Higgs boson (Higgsino) are accessible. Events are examined in which there are two photons forming a Higgs boson candidate, and at least two $b$-quark jets. In $19.7 \mathrm{fb}^{-1}$ of proton-proton collision data at $\sqrt{s}=8 \mathrm{TeV}$, recorded in the CMS experiment, no evidence of a signal is found and lower limits at the 95\% confidence level are set, excluding the top squark mass below 360 to $410 \mathrm{GeV}$, depending on the Higgsino mass.
\end{abstract}

DOI: 10.1103/PhysRevLett.112.161802

PACS numbers: 14.80.Ly, 12.60.Jv, 14.80.Da

The recent measurements of the properties of the new boson with mass around $126 \mathrm{GeV}$ by ATLAS [1,2] and CMS [3,4] experiments confirm that it is consistent with the standard model (SM) Higgs boson. One of the most important problems in particle physics is now to understand why the boson's mass is so small compared to the Planck scale. Supersymmetry (SUSY) offers an elegant solution to this hierarchy problem. Although experimental searches have found no signs of it so far and the simplest SUSY models have become increasingly more constrained, there remain very large yet unprobed areas of SUSY parameter space that can still be "natural," i.e., have a fairly small amount of fine tuning.

The essential requirement of natural SUSY is that the masses of the superpartners of the top quark and the Higgs bosons, the top squark and the Higgsinos, be light (see, e.g., $[5,6])$. This Letter describes a search we performed for events with a topology motivated by SUSY with a gauge mediated SUSY breaking (GMSB) model [7-10]. Complete GMSB models are considered with a minimal number of accessible SUSY partners to satisfy the naturalness requirements, namely, the right-handed top squark and Higgsinos.

The lightest chargino $\left(\tilde{\chi}_{1}^{+}\right)$and neutralinos $\left(\tilde{\chi}_{1}^{0}, \tilde{\chi}_{2}^{0}\right)$ in this case are almost pure Higgsinos and are therefore almost mass degenerate.

Pairs of Higgsinos are produced either directly through electroweak production or through right-handed top squark-antisquark pairs (strong production) with cascade decays

$$
\tilde{t}_{R} \rightarrow b \tilde{\chi}_{1}^{+} \quad \text { or } \quad \tilde{t}_{R} \rightarrow t \tilde{\chi}_{i}^{0}
$$

* Full author list given at the end of the article.

Published by the American Physical Society under the terms of the Creative Commons Attribution 3.0 License. Further distribution of this work must maintain attribution to the author(s) and the published articles title, journal citation, and DOI. where $i=1,2$. The $\tilde{\chi}_{1}^{+}\left(\tilde{\chi}_{2}^{0}\right)$ subsequently decays into a very off-shell $W^{+}(Z)$ boson and a $\tilde{\chi}_{1}^{0}$. (Here, and in what follows, the charge-conjugate final states are also implied.) The near mass degeneracy of the Higgsino states requires the off-shell boson decay products to be very soft.

The decay of the lightest neutralino in all cascades is

$$
\tilde{\chi}_{1}^{0} \rightarrow H \tilde{G} \quad \text { or } \quad \tilde{\chi}_{1}^{0} \rightarrow Z \tilde{G},
$$

where $\tilde{G}$ is the gravitino. The branching fractions of $\tilde{\chi}_{1}^{0}$ decays depend on SUSY parameters. For a significant portion of the parameter space, neutralino decays into Higgs bosons dominate. This includes regions of low $\tan \beta$, the ratio of the vacuum expectation values of the two SUSY Higgs doublets, and negative values of the Higgsino mass term $\mu$ [11].

The final state of interest, therefore, has two Higgs bosons, some missing energy from the gravitinos escaping detection, and, in the case of strong production, two bottom (or top, if kinematically allowed) quarks from top squark decays, as shown in Fig. 1. SM backgrounds, e.g., from top quark pair production, are suppressed by taking advantage of the known Higgs boson mass and by considering the case when at least one of the Higgs bosons decays into two photons and at least two $b$ quarks are present from either Higgs or top squark decays. This approach also allows us to use diphoton mass sidebands for an estimate of the background from data, without sensitivity to the exact composition of the background, which is dominated by QCD production of $\gamma \gamma b \bar{b}$ events and $\gamma b \bar{b}+j$ events with the jet being misidentified as a photon.

We use a data sample of $19.7 \mathrm{fb}^{-1}$ collected during the LHC $8 \mathrm{TeV}$ running with the CMS detector, which is described in detail elsewhere [12]. The detector comprises a silicon pixel and strip tracker covering the pseudorapidity range $|\eta|<2.4$ (where $\eta=-\ln [\tan (\theta / 2)]$ and $\theta$ is the polar angle of the particle with respect to the counterclockwise proton beam direction) and electromagnetic and hadronic calorimeters, which cover $|\eta|<3.0$ and $|\eta|<5$.0, 

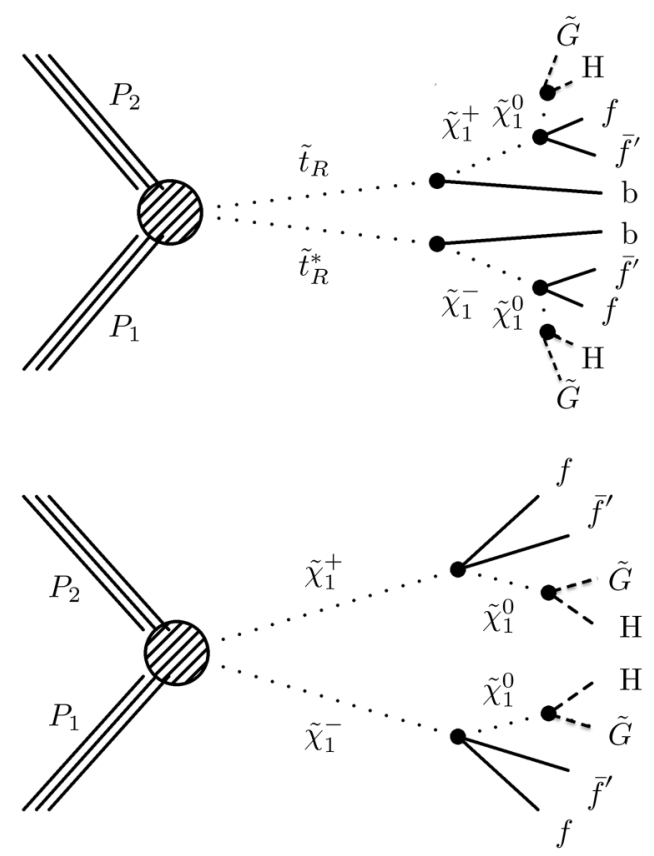

FIG. 1. Example Feynman diagram of strong (top) and electroweak (bottom) Higgsino production. The jets or leptons resulting from transitions between Higgsinos are extremely soft in both diagrams due to the near mass degeneracy among the Higgsinos.

respectively. The tracking and calorimeter systems are housed inside a 3.8 T solenoidal magnet. Muon chambers are imbedded in the iron return yoke of the solenoid up to pseudorapidity of $|\eta|<2.4$. Events are required to pass a suite of diphoton triggers that impose mild shower shape and isolation requirements and demand transverse energies $\left(E_{\mathrm{T}}=E \cdot \sin \theta\right)$ above 36 and $22 \mathrm{GeV}$ for the leading and the subleading photon, respectively.

The signal is simulated for a grid of Higgsino and top squark masses using the MADGRAPH 5 v.1.5.4 [13] and PYTHIA 6.4.22 [14] generators and fast simulation of the CMS detector [15]. The next-to-leading order (NLO) cross sections (shown in Fig. 2) are calculated using PROSPINO 2.1 [16-21]. The Higgsino mass splittings $m_{\tilde{\chi}_{2}^{0}}-m_{\tilde{\chi}_{1}^{+}}$and $m_{\tilde{\chi}_{1}^{+}}-m_{\tilde{\chi}_{1}^{0}}$ are set to $5 \mathrm{GeV}$, and a $100 \%$ branching fraction is assumed for the $\tilde{\chi}_{1}^{0} \rightarrow H \tilde{G}$ decay. All other superpartners have masses too heavy to influence SUSY production at $8 \mathrm{TeV}$.

Photon candidates are reconstructed from the energy deposits in the electromagnetic calorimeter by grouping the calorimeter's data channels into superclusters [22,23]. Photons are required to register in the barrel portion of the calorimeter $(|\eta|<1.4442)$ and to pass shower shape and isolation requirements. To reject electrons misidentified as photons, photons are also required not to have associated hit patterns in the pixel detector consistent with a track. Photon energies are calculated using multivariate regression $[4,23]$.

The particle-flow (PF) algorithm [24] is used to reconstruct individual particles (PF candidates) in the events,

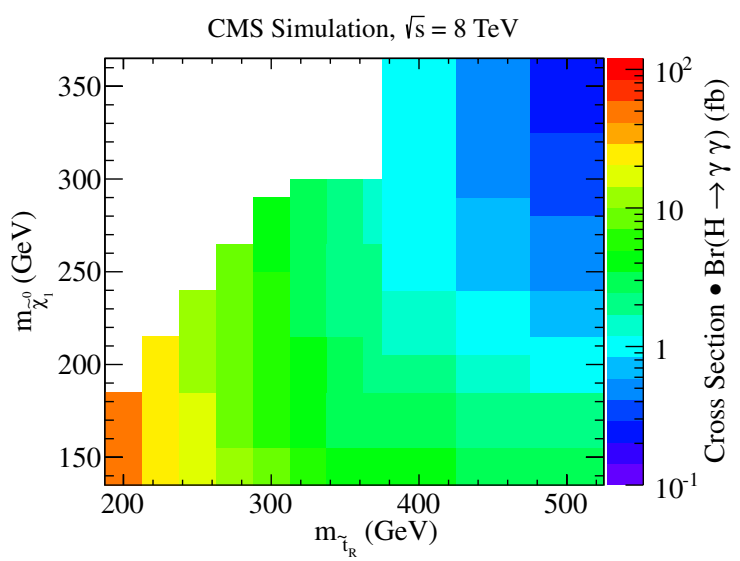

FIG. 2 (color online). Cross section times branching ratio $\operatorname{Br}(H \rightarrow \gamma \gamma)$ as a function of top squark and Higgsino masses.

combining all available subdetector information in a coherent and optimal manner. Jets are reconstructed from the PF particles using the anti- $k_{\mathrm{T}}$ [25] algorithm with a distance parameter of 0.5 and are required not to overlap with the two leading photons by imposing a requirement $\Delta R>0.6$, where $\Delta R=\sqrt{(\Delta \phi)^{2}+(\Delta \eta)^{2}}$ and $\phi$ is the azimuthal angle. Transverse momentum and $\eta$-dependent corrections to the jet energies are applied to account for residual effects of nonuniform detector response. The pileup contribution is estimated and subtracted from the jet energy using the jet area method [26] on an event-byevent basis. The combined secondary vertex (CSV) algorithm [27] is employed to identify jets that originate from a bottom quark.

The missing transverse energy $(\mathrm{MeV})$ of the event is computed as the magnitude of the vectorial sum of the transverse momenta of all PF candidates [24].

Events are selected if they have at least two identified photons with transverse energies above 40 and $25 \mathrm{GeV}$ for the leading and subleading photons, respectively. Events are also required to have at least two jets with transverse energy above $30 \mathrm{GeV}$ and $|\eta|<2.4$ and satisfying the loose (CSV-loose) $b$-tag requirements, at least one of which also must pass the medium (CSV-medium) requirement. The CSV-loose and CSV-medium working points have signal efficiencies of $80 \%-85 \%$ and $50 \%-75 \%$, respectively, and mistag rates from light-flavor jets of $8 \%-13 \%$ and $1 \%-2 \%$, respectively [27].

The events with diphoton mass between 120 and $131 \mathrm{GeV}$ constitute the signal sample, while the events with mass between 103 and $118 \mathrm{GeV}$ and between 133 and $163 \mathrm{GeV}$ compose the lower sideband and upper sideband samples, respectively.

In the investigated SUSY signal, $b$-quark jets can originate from decays of the other Higgs bosons and from decays of top squark. This is exploited by separating events into three categories with different expected backgrounds as follows: (i) events with at least one additional CSV-loose 
$b$-quark jet in addition to the two (i.e., events with three or more $b$-quark jets), (ii) events for which the invariant mass of the two $b$-quark jets is within a Higgs mass window from 95 to $155 \mathrm{GeV}$, and (iii) all other events.

The distribution of signal events among the three categories depends on the top squark and Higgsino masses. For small top squark-Higgsino mass differences, most of the signal populates category (ii), while for large mass differences categories (i) and (iii) dominate.

The search is performed independently in the three categories and the results are combined, leading to as much as 35\% improvement in expected SUSY cross section limits compared to the analysis without categorization.

The peaking background from the SM Higgs boson production with subsequent diphoton decay is found negligible using MC simulation. The diphoton mass sidebands are used to derive an estimate of all other SM background processes from data.

The diphoton distribution is fitted using a power law from 103 to $163 \mathrm{GeV}$ [28]. The region from 118 to $133 \mathrm{GeV}$, corresponding to the signal region plus the $2 \mathrm{GeV}$ "buffers" on each side of the signal region, is excluded from the fitter's consideration to prevent a potential signal from affecting the background estimate. The result of the fit is used to calculate the normalization of the background. The effects of various other fit functions have been explored, and the resulting changes in the estimated background yields are well within the uncertainty in the fit function integral from the power law fit. The fit and background estimates are done independently in all three event categories.

Binned background distributions of relevant kinematic variables are obtained for each event category separately from the lower and upper sidebands and are weighted according to the fit function integrals. This provides two independent estimates of the distribution of the SM background in the corresponding variable. No significant correlations are observed between the diphoton mass and other kinematic variables. However, to account for possible correlations, the background yield is conservatively assumed to be bounded by the independent background estimates from the upper and lower sidebands. The background prediction using this bound as a systematic uncertainty is calculated as follows. The main background estimate in each bin of a kinematic variable is formed by taking an average of the two background estimates from the sidebands. Half the difference between the two estimates is taken as a systematic uncertainty for that bin, so that the central value and its uncertainty spans the range between the two estimates coming from the lower and upper sidebands. For the main variable used in this analysis, $E_{\mathrm{T}}^{\text {miss }}$, the background uncertainty is dominated by the statistical uncertainty in the sideband samples. The small correlation between the bin errors, originating from the common normalization from the fit, is taken into account during the statistical analysis described below.
The observed distributions of $\mathrm{MeV}$ are shown in Fig. 3 separately for the three event categories, together with the background estimates from the above procedure and the expected distributions from three characteristic signal points. Table I shows the expected signal yields, background
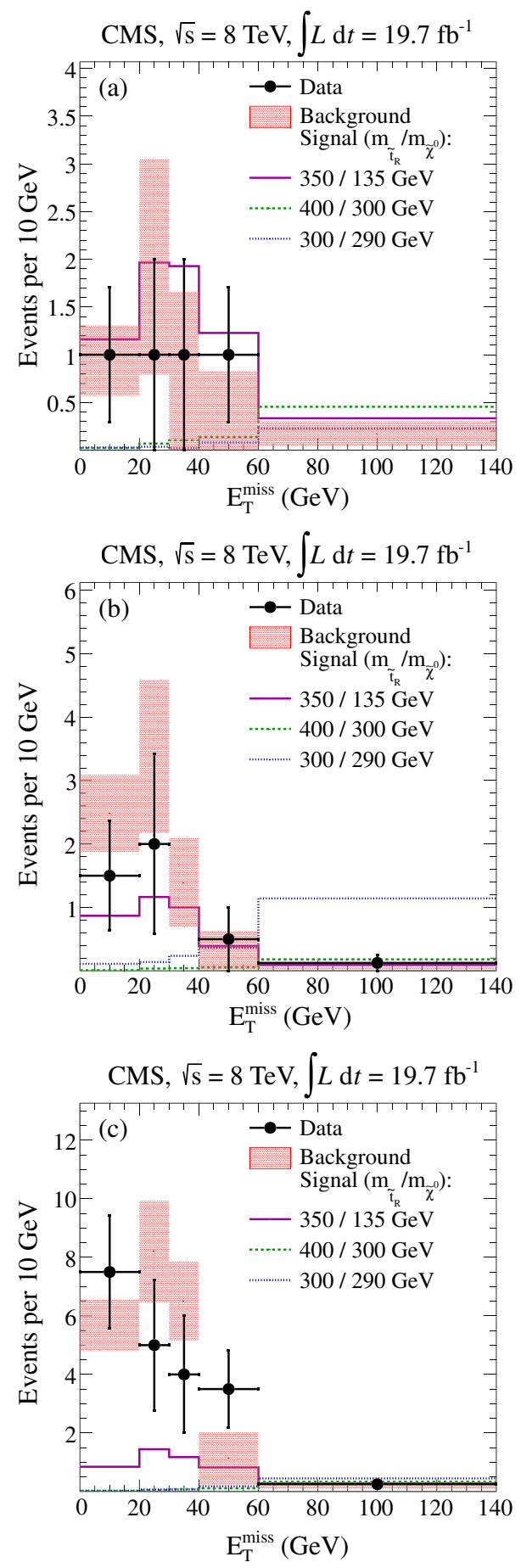

FIG. 3 (color online). $E_{\mathrm{T}}^{\text {miss }}$ distributions for the data, background predictions, and representative signals for the three event categories (i), (ii), and (iii) in frames (a), (b), and (c), respectively. For each histogram, the last bin includes the overflow. 
TABLE I. Expected and observed event counts. The yields for three signal points are shown for comparison indicating $m_{\tilde{t}_{R}}$ and $m_{\tilde{\chi}_{1}^{0}}$ in $\mathrm{GeV}$, respectively.

\begin{tabular}{lccc}
\hline \hline Category & (i) & (ii) & (iii) \\
\hline Signal 350/135 & 10.7 & 2.0 & 6.8 \\
Signal 300/290 & 2.1 & 10.1 & 3.9 \\
Signal 400/300 & 4.0 & 1.4 & 2.8 \\
Expected background & $6.7 \pm 1.4$ & $10.5 \pm 1.8$ & $29.7 \pm 2.8$ \\
Observed & 6 & 7 & 33 \\
\hline \hline
\end{tabular}

predictions, and observed event counts for the three event categories. The observations are in agreement with the background predictions.

Since the data agree with the expected background, we proceed to set limits on the SUSY model described above. The expected limits were calculated using a variety of kinematic variables and it was determined that $E_{\mathrm{T}}^{\text {miss }}$ is the single most sensitive variable.

The observed $E_{\mathrm{T}}^{\text {miss }}$ distributions for the three event categories, shown in Fig. 3, together with the signal and background expectations listed in Table I, are used as input to the limit setting procedure.

A frequentist LHC-style profiled likelihood test statistic is used [29-31]. For each model mass point, the modified frequentist $\mathrm{CL}_{\mathrm{S}}$ method is used to calculate upper limits on the cross section for the model.

The dominant uncertainty in the analysis is the statistical uncertainty of the background prediction. The leading sources of systematic uncertainties are the $b$-quark jet identification efficiency and the jet energy scale. The magnitude of the uncertainties depends on the event category and $E_{\mathrm{T}}^{\text {miss }}$. For $b$-quark jet identification it varies from $1 \%$ to $5 \%$ for categories with two $b$-quark jets and from $6 \%$ to $17 \%$ for the three or more $b$-quark jets. For the jet energy scale, the uncertainty is important only for $E_{\mathrm{T}}^{\text {miss }}$ values below $40 \mathrm{GeV}$ and varies from $7 \%$ to $43 \%$.

Other sources of systematic uncertainty in the expected signal yield include the uncertainties in integrated luminosity $(2.6 \%)$ [32], the diphoton trigger efficiency $(0.1 \%)$, the photon reconstruction and identification efficiency $(1 \%)$, and the photon resolution uncertainty (1\%) [4]. All systematic uncertainties are correlated among the event categories and are treated as nuisance parameters in the likelihood, profiled according to their estimated value.

Figure 4 shows the limits on the GMSB model in the top squark-Higgsino mass plane. Depending on the Higgsino mass, and conservatively using the -1 standard deviation value for the theoretical cross section [16], top squark masses below 360 to $410 \mathrm{GeV}$ are excluded at a $95 \%$ confidence level, corresponding to the region to the left of the leftmost thin dashed black line in Fig. 4.

To summarize, a search for a natural SUSY scenario with light Higgsinos and a top squark was performed, using Higgs tagging in the diphoton decay mode, in a final state containing at least two photons and two or more $b$-quark jets.

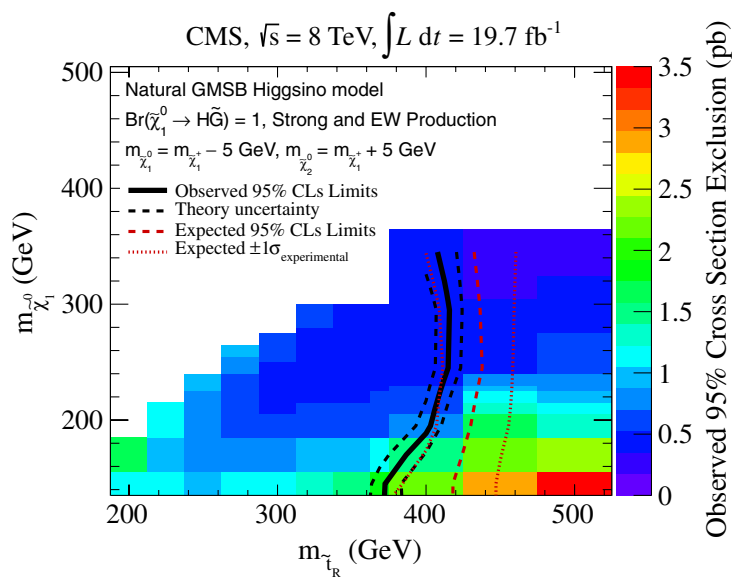

FIG. 4 (color online). Limits on SUSY production cross sections for different top squark and Higgsino masses. The regions to the left of the contours are expected (thick dashed red or gray curves) and observed (solid black curves) to be excluded at the $95 \%$ level. Black dashed curves reflect the theoretical uncertainty on the cross section. Red dotted curves correspond to 1 standard deviation in expected sensitivity.

No evidence for a signal is observed, and $95 \%$ confidence level limits are set in the top squark-Higgsino mass plane, excluding a significant portion of the natural parameter space up to top squark masses of $410 \mathrm{GeV}$.

We congratulate our colleagues in the CERN accelerator departments for the excellent performance of the LHC and thank the technical and administrative staffs at CERN and at other CMS institutes for their contributions to the success of the CMS effort. In addition, we gratefully acknowledge the computing centers and personnel of the Worldwide LHC Computing Grid for delivering so effectively the computing infrastructure essential to our analyses. Finally, we acknowledge the enduring support for the construction and operation of the LHC and the CMS detector provided by the following funding agencies: BMWF and FWF (Austria); FNRS and FWO (Belgium); CNPq, CAPES, FAPERJ, and FAPESP (Brazil); MES (Bulgaria); CERN; CAS, MoST, and NSFC (China); COLCIENCIAS (Colombia); MSES (Croatia); RPF (Cyprus); MoER, SF0690030s09 and ERDF (Estonia); Academy of Finland, MEC, and HIP (Finland); CEA and CNRS/ IN2P3 (France); BMBF, DFG, and HGF (Germany); GSRT (Greece); OTKA and NIH (Hungary); DAE and DST (India); IPM (Iran); SFI (Ireland); INFN (Italy); NRF and WCU (Republic of Korea); LAS (Lithuania); CINVESTAV, CONACYT, SEP, and UASLP-FAI (Mexico); MBIE (New Zealand); PAEC (Pakistan); MSHE and NSC (Poland); FCT (Portugal); JINR (Dubna); MON, RosAtom, RAS and RFBR (Russia); MESTD (Serbia); SEIDI and CPAN (Spain); Swiss Funding Agencies (Switzerland); NSC (Taipei); ThEPCenter, IPST, STAR and NSTDA (Thailand); TUBITAK and TAEK (Turkey); NASU (Ukraine); STFC (United Kingdom); DOE and NSF (USA). 
[1] ATLAS Collaboration, Phys. Lett. B 726, 88 (2013).

[2] ATLAS Collaboration, Phys. Lett. B 726, 120 (2013).

[3] CMS Collaboration, Phys. Rev. Lett. 110, 081803 (2013).

[4] CMS Collaboration, J. High Energy Phys. 06 (2013) 081.

[5] R. Barbieri and G. F. Giudice, Nucl. Phys. B306, 63 (1988).

[6] M. Papucci, J. T. Ruderman, and A. Weiler, J. High Energy Phys. 09 (2012) 035.

[7] S. Dimopoulos, M. Dine, S. Raby, and S. D. Thomas, Phys. Rev. Lett. 76, 3494 (1996).

[8] S. Ambrosanio, G. L. Kane, G. D. Kribs, S. P. Martin, and S. Mrenna, Phys. Rev. D 54, 5395 (1996).

[9] K. T. Matchev and S. D. Thomas, Phys. Rev. D 62, 077702 (2000).

[10] K. Howe and P. Saraswat, J. High Energy Phys. 10 (2012) 065.

[11] P. Meade, M. Reece, and D. Shih, J. High Energy Phys. 05 (2010) 105.

[12] CMS Collaboration, JINST 3, S08004 (2008).

[13] J. Alwall, M. Herquet, F. Maltoni, O. Mattelaer, and T. Stelzer, J. High Energy Phys. 06 (2011) 128.

[14] T. Sjöstrand, S. Mrenna, and P. Z. Skands, J. High Energy Phys. 05 (2006) 026.

[15] CMS Collaboration, J. Phys. Conf. Ser. 331, 032049 (2011).

[16] M. Krämer, A. Kulesza, R. van der Leeuw, M. Mangano, S. Padhi, T. Plehn, and X. Portell, arXiv:1206.2892.

[17] W. Beenakker, R. Hopker, M. Spira, and P. M. Zerwas, Nucl. Phys. B492, 51 (1997).

[18] A. Kulesza and L. Motyka, Phys. Rev. Lett. 102, 111802 (2009).

[19] W. Beenakker, S. Brensing, M. Krämer, A. Kulesza, E. Laenen, and I. Niessen, J. High Energy Phys. 12 (2009) 041.
[20] W. Beenakker, S. Brensing, M. Krämer, A. Kulesza, E. Laenen, L. Motyka, and I. Niessen, Int. J. Mod. Phys. A 26, 2637 (2011).

[21] W. Beenakker, R. Hopker, and M. Spira, arXiv:hep-ph/ 9611232.

[22] CMS Collaboration, CMS Physics Analysis Summary, Report No. CMS-PAS-EGM-10-005, 2010, http://cdsweb .cern.ch/record/1279143.

[23] CMS Collaboration, JINST 8, P09009 (2013).

[24] CMS Collaboration, CMS Physics Analysis Summary, Report No. CMS-PAS-PFT-10-002, 2010, http://cdsweb .cern.ch/record/1279341.

[25] M. Cacciari, G. P. Salam, and G. Soyez, J. High Energy Phys. 04 (2008), 063.

[26] M. Cacciari and G. P. Salam, Phys. Lett. B 659, 119 (2008).

[27] CMS Collaboration, JINST 8, P04013 (2013).

[28] See Supplemental Material at http://link.aps.org/ supplemental/10.1103/PhysRevLett.112.161802 for the fits of diphoton mass distributions and distributions of considered kinematic variables.

[29] ATLAS Collaboration, CMS Collaboration, and LHC Higgs Combination Group, Techncial Report No. CMS-NOTE2011-005, ATL-PHYS-PUB-2011-11, CERN, Geneva, 2011, http://cds.cern.ch/record/1375842/files/ATL-PHYSPUB-2011-011.pdf.

[30] T. Junk, Nucl. Instrum. Methods Phys. Res., Sect. A 434, 435 (1999).

[31] A. L. Read, J. Phys. G 28, 2693 (2002).

[32] CMS Collaboration, CMS Physics Analysis Summary, Report No. CMS-PAS-LUM-13-001, 2013, http://cds.cern .ch/record/1598864/files/LUM-13-001-pas.pdf.

S. Chatrchyan, ${ }^{1}$ V. Khachatryan, ${ }^{1}$ A. M. Sirunyan, ${ }^{1}$ A. Tumasyan, ${ }^{1}$ W. Adam, ${ }^{2}$ T. Bergauer, ${ }^{2}$ M. Dragicevic, ${ }^{2}$ J. Erö, ${ }^{2}$ C. Fabjan, ${ }^{2 b}$ M. Friedl, ${ }^{2}$ R. Frühwirth, ${ }^{2 b}$ V. M. Ghete, ${ }^{2}$ C. Hartl, ${ }^{2}$ N. Hörmann, ${ }^{2}$ J. Hrubec, ${ }^{2}$ M. Jeitler, ${ }^{2 b}$ W. Kiesenhofer, ${ }^{2}$ V. Knünz, ${ }^{2}$ M. Krammer, ${ }^{2 b}$ I. Krätschmer, ${ }^{2}$ D. Liko, ${ }^{2}$ I. Mikulec, ${ }^{2}$ D. Rabady, ${ }^{2 \mathrm{c}}$ B. Rahbaran, ${ }^{2}$ H. Rohringer, ${ }^{2}$ R. Schöfbeck, ${ }^{2}$ J. Strauss, ${ }^{2}$ A. Taurok, ${ }^{2}$ W. Treberer-Treberspurg, ${ }^{2}$ W. Waltenberger, ${ }^{2}$ C.-E. Wulz,${ }^{2 b}$ V. Mossolov,${ }^{3}$ N. Shumeiko, ${ }^{3}$ J. Suarez Gonzalez, ${ }^{3}$ S. Alderweireldt, ${ }^{4}$ M. Bansal, ${ }^{4}$ S. Bansal, ${ }^{4}$ T. Cornelis, ${ }^{4}$ E. A. De Wolf, ${ }^{4}$ X. Janssen, ${ }^{4}$ A. Knutsson, ${ }^{4}$ S. Luyckx, ${ }^{4}$ L. Mucibello, ${ }^{4}$ S. Ochesanu, ${ }^{4}$ B. Roland, ${ }^{4}$ R. Rougny, ${ }^{4}$ H. Van Haevermaet, ${ }^{4}$ P. Van Mechelen, ${ }^{4}$ N. Van Remortel, ${ }^{4}$ A. Van Spilbeeck, ${ }^{4}$ F. Blekman, ${ }^{5}$ S. Blyweert, ${ }^{5}$ J. D'Hondt, ${ }^{5}$ N. Heracleous, ${ }^{5}$ A. Kalogeropoulos, ${ }^{5}$ J. Keaveney, ${ }^{5}$ T. J. Kim, ${ }^{5}$ S. Lowette, ${ }^{5}$ M. Maes, ${ }^{5}$ A. Olbrechts, ${ }^{5}$ D. Strom, ${ }^{5}$ S. Tavernier, ${ }^{5}$ W. Van Doninck, ${ }^{5}$ P. Van Mulders, ${ }^{5}$ G. P. Van Onsem, ${ }^{5}$ I. Villella, ${ }^{5}$ C. Caillol, ${ }^{6}$ B. Clerbaux,${ }^{6}$ G. De Lentdecker, ${ }^{6}$ L. Favart, ${ }^{6}$ A. P. R. Gay, ${ }^{6}$ A. Léonard, ${ }^{6}$ P. E. Marage, ${ }^{6}$ A. Mohammadi, ${ }^{6}$ L. Perniè, ${ }^{6}$ T. Reis, ${ }^{6}$ T. Seva,${ }^{6}$ L. Thomas, ${ }^{6}$ C. Vander Velde, ${ }^{6}$ P. Vanlaer, ${ }^{6}$ J. Wang, ${ }^{6}$ V. Adler, ${ }^{7}$ K. Beernaert, ${ }^{7}$ L. Benucci, ${ }^{7}$ A. Cimmino, ${ }^{7}$ S. Costantini, ${ }^{7}$ S. Dildick, ${ }^{7}$ G. Garcia, ${ }^{7}$ B. Klein, ${ }^{7}$ J. Lellouch, ${ }^{7}$ J. Mccartin, ${ }^{7}$ A. A. Ocampo Rios, ${ }^{7}$ D. Ryckbosch, ${ }^{7}$ S. Salva Diblen, ${ }^{7}$ M. Sigamani, ${ }^{7}$ N. Strobbe, ${ }^{7}$ F. Thyssen, ${ }^{7}$ M. Tytgat, ${ }^{7}$ S. Walsh, ${ }^{7}$ E. Yazgan,${ }^{7}$ N. Zaganidis, ${ }^{7}$ S. Basegmez,${ }^{8}$ C. Beluffi,${ }^{8 d}$ G. Bruno,${ }^{8}$ R. Castello,${ }^{8}$ A. Caudron, ${ }^{8}$ L. Ceard, ${ }^{8}$ G. G. Da Silveira, ${ }^{8}$ C. Delaere, ${ }^{8}$ T. du Pree, ${ }^{8}$ D. Favart, ${ }^{8}$ L. Forthomme, ${ }^{8}$ A. Giammanco,${ }^{8 \mathrm{e}}$ J. Hollar, ${ }^{8}$ P. Jez, ${ }^{8}$ M. Komm, ${ }^{8}$ V. Lemaitre,${ }^{8}$ J. Liao, ${ }^{8}$ O. Militaru, ${ }^{8}$ C. Nuttens, ${ }^{8}$ D. Pagano, ${ }^{8}$ A. Pin, ${ }^{8}$ K. Piotrzkowski, ${ }^{8}$ A. Popov,${ }^{8 f}$ L. Quertenmont,${ }^{8}$ M. Selvaggi, ${ }^{8}$ M. Vidal Marono, ${ }^{8}$ J. M. Vizan Garcia, ${ }^{8}$ N. Beliy, ${ }^{9}$ T. Caebergs, ${ }^{9}$ E. Daubie, ${ }^{9}$ G. H. Hammad, ${ }^{9}$ G. A. Alves, ${ }^{10}$ M. Correa Martins Junior, ${ }^{10}$ T. Martins, ${ }^{10}$ M. E. Pol, ${ }^{10}$ M. H. G. Souza, ${ }^{10}$ W. L. Aldá Júnior, ${ }^{11}$ W. Carvalho, ${ }^{11}$ J. Chinellato, ${ }^{11 g}$ A. Custódio, ${ }^{11}$ E. M. Da Costa, ${ }^{11}$ D. De Jesus Damiao, ${ }^{11}$ C. De Oliveira Martins, ${ }^{11}$ S. Fonseca De Souza, ${ }^{11}$ H. Malbouisson, ${ }^{11}$ M. Malek, ${ }^{11}$ D. Matos Figueiredo, ${ }^{11}$ L. Mundim, ${ }^{11}$ H. Nogima, ${ }^{11}$ W. L. Prado Da Silva,${ }^{11}$ J. Santaolalla, ${ }^{11}$ A. Santoro, ${ }^{11}$ A. Sznajder,${ }^{11}$ E. J. Tonelli Manganote, ${ }^{1 \mathrm{~g}}$ A. Vilela Pereira, ${ }^{11}$ C. A. Bernardes, ${ }^{12 b}$ F. A. Dias, ${ }^{12 \mathrm{ah}}$ T. R. Fernandez Perez Tomei, ${ }^{12 \mathrm{a}}$ 
E. M. Gregores, ${ }^{12 b}$ C. Lagana, ${ }^{12 a}$ P. G. Mercadante, ${ }^{12 b}$ S. F. Novaes, ${ }^{12 a}$ S. S. Padula, ${ }^{12 a}$ V. Genchev, ${ }^{13 c}$ P. Iaydjiev, ${ }^{13 c}$ A. Marinov, ${ }^{13}$ S. Piperov, ${ }^{13}$ M. Rodozov ${ }^{13}$ G. Sultanov, ${ }^{13}$ M. Vutova, ${ }^{13}$ A. Dimitrov, ${ }^{14}$ I. Glushkov, ${ }^{14}$ R. Hadjiiska, ${ }^{14}$ V. Kozhuharov, ${ }^{14}$ L. Litov, ${ }^{14}$ B. Pavlov, ${ }^{14}$ P. Petkov, ${ }^{14}$ J. G. Bian, ${ }^{15}$ G. M. Chen, ${ }^{15}$ H. S. Chen, ${ }^{15}$ M. Chen, ${ }^{15}$ R. Du, ${ }^{15}$ C. H. Jiang, ${ }^{15}$ D. Liang, ${ }^{15}$ S. Liang, ${ }^{15}$ X. Meng,${ }^{15}$ R. Plestina, ${ }^{15 i}$ J. Tao, ${ }^{15}$ X. Wang, ${ }^{15}$ Z. Wang,,${ }^{15}$ C. Asawatangtrakuldee,${ }^{16}$ Y. Ban, ${ }^{16}$ Y. Guo, ${ }^{16}$ Q. Li, ${ }^{16}$ W. Li, ${ }^{16}$ S. Liu, ${ }^{16}$ Y. Mao, ${ }^{16}$ S. J. Qian, ${ }^{16}$ D. Wang, ${ }^{16}$ L. Zhang, ${ }^{16}$ W. Zou, ${ }^{16}$ C. Avila, ${ }^{17}$ C. A. Carrillo Montoya, ${ }^{17}$ L. F. Chaparro Sierra ${ }^{17}$ C. Florez,${ }^{17}$ J. P. Gomez, ${ }^{17}$ B. Gomez Moreno, ${ }^{17}$ J. C. Sanabria, ${ }^{17}$ N. Godinovic, ${ }^{18}$ D. Lelas, ${ }^{18}$ D. Polic,${ }^{18}$ I. Puljak, ${ }^{18}$ Z. Antunovic, ${ }^{19}$ M. Kovac, ${ }^{19}$ V. Brigljevic,${ }^{20}$ K. Kadija,${ }^{20}$ J. Luetic, ${ }^{20}$ D. Mekterovic, ${ }^{20}$ S. Morovic ${ }^{20}$ L. Tikvica, ${ }^{20}$ A. Attikis, ${ }^{21}$ G. Mavromanolakis, ${ }^{21}$ J. Mousa, ${ }^{21}$ C. Nicolaou, ${ }^{21}$ F. Ptochos,${ }^{21}$ P. A. Razis, ${ }^{21}$ M. Finger, ${ }^{22}$ M. Finger Jr., ${ }^{22}$ A. A. Abdelalim, ${ }^{23 \mathrm{j}}$ Y. Assran, ${ }^{23 \mathrm{k}}$ S. Elgammal, ${ }^{23 \mathrm{j}}$ A. Ellithi Kamel, ${ }^{231}$ M. A. Mahmoud, ${ }^{23 m}$ A. Radi, ${ }^{23, o, p}$ M. Kadastik, ${ }^{24}$ M. Müntel, ${ }^{24}$ M. Murumaa, ${ }^{24}$ M. Raidal, ${ }^{24}$ L. Rebane,${ }^{24}$ A. Tiko, ${ }^{24}$ P. Eerola, ${ }^{25}$ G. Fedii, ${ }^{25}$ M. Voutilainen, ${ }^{25}$ J. Härkönen, ${ }^{26}$ V. Karimäki, ${ }^{26}$ R. Kinnunen, ${ }^{26}$ M. J. Kortelainen, ${ }^{26}$ T. Lampén, ${ }^{26}$ K. Lassila-Perini, ${ }^{26}$ S. Lehti, ${ }^{26}$ T. Lindén, ${ }^{26}$ P. Luukka, ${ }^{26}$ T. Mäenpää, ${ }^{26}$ T. Peltola, ${ }^{26}$ E. Tuominen, ${ }^{26}$ J. Tuominiemi, ${ }^{26}$ E. Tuovinen ${ }^{26}$ L. Wendland, ${ }^{26}$ T. Tuuva, ${ }^{27}$ M. Besancon, ${ }^{28}$ F. Couderc, ${ }^{28}$ M. Dejardin, ${ }^{28}$ D. Denegri, ${ }^{28}$ B. Fabbro, ${ }^{28}$ J. L. Faure, ${ }^{28}$ F. Ferri, ${ }^{28}$ S. Ganjour, ${ }^{28}$ A. Givernaud, ${ }^{28}$ P. Gras, ${ }^{28}$ G. Hamel de Monchenault, ${ }^{28}$ P. Jarry, ${ }^{28}$ E. Locci, ${ }^{28}$ J. Malcles, ${ }^{28}$ A. Nayak, ${ }^{28}$ J. Rander, ${ }^{28}$ A. Rosowsky, ${ }^{28}$ M. Titov ${ }^{28}$ S. Baffioni, ${ }^{29}$ F. Beaudette, ${ }^{29}$ P. Busson, ${ }^{29}$ C. Charlot,${ }^{29}$ N. Daci, ${ }^{29}$ T. Dahms,${ }^{29}$ M. Dalchenko, ${ }^{29}$ L. Dobrzynski, ${ }^{29}$ A. Florent, ${ }^{29}$ R. Granier de Cassagnac, ${ }^{29}$ P. Miné,${ }^{29}$ C. Mironov, ${ }^{29}$ I. N. Naranjo, ${ }^{29}$ M. Nguyen, ${ }^{29}$ C. Ochando, ${ }^{29}$ P. Paganini, ${ }^{29}$ D. Sabes,${ }^{29}$ R. Salerno,${ }^{29}$ Y. Sirois,${ }^{29}$ C. Veelken, ${ }^{29}$ Y. Yilmaz ${ }^{29}$ A. Zabi, ${ }^{29}$ J.-L. Agram, ${ }^{30 p}$ J. Andrea ${ }^{30}$ D. Bloch,${ }^{30}$ J.-M. Brom,${ }^{30}$ E. C. Chabert,${ }^{30}$ C. Collard,${ }^{30}$ E. Conte,${ }^{30 p}$ F. Drouhin, ${ }^{30 p}$ J.-C. Fontaine, ${ }^{30 \mathrm{p}}$ D. Gelé, ${ }^{30}$ U. Goerlach,${ }^{30}$ C. Goetzmann, ${ }^{30}$ P. Juillot, ${ }^{30}$ A.-C. Le Bihan, ${ }^{30}$ P. Van Hove, ${ }^{30}$ S. Gadrat, ${ }^{31}$ S. Beauceron, ${ }^{32}$ N. Beaupere, ${ }^{32}$ G. Boudoul, ${ }^{32}$ S. Brochet,${ }^{32}$ J. Chasserat,${ }^{32}$ R. Chierici, ${ }^{32}$ D. Contardo,${ }^{32 c}$ P. Depasse, ${ }^{32}$ H. El Mamouni, ${ }^{32}$ J. Fan, ${ }^{32}$ J. Fay, ${ }^{32}$ S. Gascon, ${ }^{32}$ M. Gouzevitch, ${ }^{32}$ B. Ille, ${ }^{32}$ T. Kurca, ${ }^{32}$ M. Lethuillier, ${ }^{32}$ L. Mirabito, ${ }^{32}$ S. Perries, ${ }^{32}$ J. D. Ruiz Alvarez, ${ }^{32}$ L. Sgandurra, ${ }^{32}$ V. Sordini, ${ }^{32}$ M. Vander Donckt, ${ }^{32}$ P. Verdier, ${ }^{32}$ S. Viret, ${ }^{32}$ H. Xiao, ${ }^{32}$ Z. Tsamalaidze, ${ }^{33 q}$ C. Autermann, ${ }^{34}$ S. Beranek, ${ }^{34}$ M. Bontenackels, ${ }^{34}$ B. Calpas,${ }^{34}$ M. Edelhoff, ${ }^{34}$ L. Feld,${ }^{34}$ O. Hindrichs,${ }^{34}$ K. Klein, ${ }^{34}$ A. Ostapchuk, ${ }^{34}$ A. Perieanu,${ }^{34}$ F. Raupach, ${ }^{34}$ J. Sammet, ${ }^{34}$ S. Schael, ${ }^{34}$ D. Sprenger,${ }^{34}$ H. Weber, ${ }^{34}$ B. Wittmer,${ }^{34}$ V. Zhukov, ${ }^{34 f}$ M. Ata, ${ }^{35}$ J. Caudron, ${ }^{35}$ E. Dietz-Laursonn, ${ }^{35}$ D. Duchardt,${ }^{35}$ M. Erdmann, ${ }^{35}$ R. Fischer, ${ }^{35}$ A. Güth, ${ }^{35}$ T. Hebbeker, ${ }^{35}$ C. Heidemann, ${ }^{35}$ K. Hoepfner, ${ }^{35}$ D. Klingebiel, ${ }^{35}$ S. Knutzen, ${ }^{35}$ P. Kreuzer, ${ }^{35}$ M. Merschmeyer, ${ }^{35}$ A. Meyer, ${ }^{35}$ M. Olschewski, ${ }^{35}$ K. Padeken, ${ }^{35}$ P. Papacz,${ }^{35}$ H. Reithler, ${ }^{35}$ S. A. Schmitz, ${ }^{35}$ L. Sonnenschein,${ }^{35}$ D. Teyssier,${ }^{35}$ S. Thüer,${ }^{35}$ M. Weber ${ }^{35}$ V. Cherepanov, ${ }^{36}$ Y. Erdogan,${ }^{36}$ G. Flügge, ${ }^{36}$ H. Geenen, ${ }^{36}$ M. Geisler, ${ }^{36}$ W. Haj Ahmad, ${ }^{36}$ F. Hoehle, ${ }^{36}$ B. Kargoll,${ }^{36}$ T. Kress,${ }^{36}$ Y. Kuessel, ${ }^{36}$ J. Lingemann, ${ }^{36 c}$ A. Nowack,${ }^{36}$ I. M. Nugent,${ }^{36}$ L. Perchalla, ${ }^{36}$ O. Pooth,${ }^{36}$ A. Stahl, ${ }^{36}$ I. Asin, ${ }^{37}$ N. Bartosik, ${ }^{37}$ J. Behr, ${ }^{37}$ W. Behrenhoff,,${ }^{37}$ U. Behrens, ${ }^{37}$ A. J. Bell, ${ }^{37}$ M. Bergholz,${ }^{37 r}$ A. Bethani,${ }^{37}$ K. Borras, ${ }^{37}$ A. Burgmeier, ${ }^{37}$ A. Cakir ${ }^{37}$ L. Calligaris, ${ }^{37}$ A. Campbell, ${ }^{37}$ S. Choudhury,${ }^{37}$ F. Costanza,${ }^{37}$ C. Diez Pardos,${ }^{37}$ S. Dooling,${ }^{37}$ T. Dorland, ${ }^{37}$ G. Eckerlin, ${ }^{37}$ D. Eckstein, ${ }^{37}$ T. Eichhorn, ${ }^{37}$ G. Flucke, ${ }^{37}$ A. Geiser, ${ }^{37}$ A. Grebenyuk, ${ }^{37}$ P. Gunnellini, ${ }^{37}$ S. Habib,${ }^{37}$ J. Hauk,${ }^{37}$ G. Hellwig, ${ }^{37}$ M. Hempel,${ }^{37}$ D. Horton, ${ }^{37}$ H. Jung,${ }^{37}$ M. Kasemann, ${ }^{37}$ P. Katsas,${ }^{37}$ J. Kieseler, ${ }^{37}$ C. Kleinwort, ${ }^{37}$ M. Krämer, ${ }^{37}$ D. Krücker, ${ }^{37}$ W. Lange, ${ }^{37}$ J. Leonard, ${ }^{37}$ K. Lipka, ${ }^{37}$ W. Lohmann,,${ }^{37 \mathrm{r}}$ B. Lutz,${ }^{37}$ R. Mankel, ${ }^{37}$ I. Marfin, ${ }^{37}$ I.-A. Melzer-Pellmann, ${ }^{37}$ A. B. Meyer,${ }^{37}$ J. Mnich, ${ }^{37}$ A. Mussgiller, ${ }^{37}$ S. Naumann-Emme, ${ }^{37}$ O. Novgorodova, ${ }^{37}$ F. Nowak, ${ }^{37}$ H. Perrey, ${ }^{37}$ A. Petrukhin, ${ }^{37}$ D. Pitzl,${ }^{37}$ R. Placakyte, ${ }^{37}$ A. Raspereza, ${ }^{37}$ P. M. Ribeiro Cipriano, ${ }^{37}$ C. Riedl, ${ }^{37}$ E. Ron, ${ }^{37}$ M. O. Sahin, ${ }^{37}$ J. Salfeld-Nebgen, ${ }^{37}$ P. Saxena, ${ }^{37}$ R. Schmidt, ${ }^{37 r}$ T. Schoerner-Sadenius, ${ }^{37}$ M. Schröder, ${ }^{37}$ M. Stein,${ }^{37}$ A. D. R. Vargas Trevino, ${ }^{37}$ R. Walsh,${ }^{37}$ C. Wissing, ${ }^{37}$ M. Aldaya Martin, ${ }^{38}$ V. Blobel,${ }^{38}$ H. Enderle, ${ }^{38}$ J. Erfle,${ }^{38}$ E. Garutti, ${ }^{38}$ K. Goebel, ${ }^{38}$ M. Görner, ${ }^{38}$ M. Gosselink, ${ }^{38}$ J. Haller, ${ }^{38}$ R. S. Höing, ${ }^{38}$ H. Kirschenmann, ${ }^{38}$ R. Klanner, ${ }^{38}$ R. Kogler, ${ }^{38}$ J. Lange, ${ }^{38}$ T. Lapsien, ${ }^{38}$ I. Marchesini, ${ }^{38}$ J. Ott, ${ }^{38}$ T. Peiffer, ${ }^{38}$ N. Pietsch, ${ }^{38}$ D. Rathjens,${ }^{38}$ C. Sander ${ }^{38}$ H. Schettler, ${ }^{38}$ P. Schleper, ${ }^{38}$ E. Schlieckau, ${ }^{38}$ A. Schmidt, ${ }^{38}$ M. Seidel,${ }^{38}$ J. Sibille, ${ }^{38 s}$ V. Sola, ${ }^{38}$ H. Stadie, ${ }^{38}$ G. Steinbrück, ${ }^{38}$ D. Troendle, ${ }^{38}$ E. Usai, ${ }^{38}$ L. Vanelderen, ${ }^{38}$ C. Barth,${ }^{39}$ C. Baus, ${ }^{39}$ J. Berger, ${ }^{39}$ C. Böser, ${ }^{39}$ E. Butz, ${ }^{39}$ T. Chwalek, ${ }^{39}$ W. De Boer, ${ }^{39}$ A. Descroix, ${ }^{39}$ A. Dierlamm, ${ }^{39}$ M. Feindt, ${ }^{39}$ M. Guthoff, ${ }^{39 c}$ F. Hartmann, ${ }^{39 c}$ T. Hauth ${ }^{39 c}$ H. Held, ${ }^{39}$

K. H. Hoffmann, ${ }^{39}$ U. Husemann, ${ }^{39}$ I. Katkov, ${ }^{39 f}$ A. Kornmayer, ${ }^{39 c}$ E. Kuznetsova, ${ }^{39}$ P. Lobelle Pardo, ${ }^{39}$ D. Martschei, ${ }^{39}$ M. U. Mozer, ${ }^{39}$ T. Müller, ${ }^{39}$ M. Niegel, ${ }^{39}$ A. Nürnberg, ${ }^{39}$ O. Oberst, ${ }^{39}$ G. Quast,${ }^{39}$ K. Rabbertz, ${ }^{39}$ F. Ratnikov, ${ }^{39}$ S. Röcker, ${ }^{39}$ F.-P. Schilling, ${ }^{39}$ G. Schott, ${ }^{39}$ H. J. Simonis, ${ }^{39}$ F. M. Stober, ${ }^{39}$ R. Ulrich, ${ }^{39}$ J. Wagner-Kuhr, ${ }^{39}$ S. Wayand, ${ }^{39}$ T. Weiler, ${ }^{39}$ R. Wolf, ${ }^{39}$ M. Zeise ${ }^{39}$ G. Anagnostou, ${ }^{40}$ G. Daskalakis, ${ }^{40}$ T. Geralis, ${ }^{40}$ S. Kesisoglou, ${ }^{40}$ A. Kyriakis, ${ }^{40}$ D. Loukas, ${ }^{40}$ A. Markou, ${ }^{40}$ C. Markou, ${ }^{40}$ E. Ntomari, ${ }^{40}$ A. Psallidas, ${ }^{40}$ I. Topsis-giotis, ${ }^{40}$ L. Gouskos, ${ }^{41}$ A. Panagiotou, ${ }^{41}$ N. Saoulidou, ${ }^{41}$ E. Stiliaris, ${ }^{41}$ X. Aslanoglou, ${ }^{42}$ I. Evangelou, ${ }^{42}$ G. Flouris, ${ }^{42}$ C. Foudas, ${ }^{42}$ J. Jones, ${ }^{42}$ P. Kokkas, ${ }^{42}$ N. Manthos, ${ }^{42}$ 
I. Papadopoulos, ${ }^{42}$ E. Paradas, ${ }^{42}$ G. Bencze,${ }^{43}$ C. Hajdu, ${ }^{43}$ P. Hidas, ${ }^{43}$ D. Horvath, ${ }^{43 t}$ F. Sikler, ${ }^{43}$ V. Veszpremi, ${ }^{43}$ G. Vesztergombi, ${ }^{43 u}$ A. J. Zsigmond, ${ }^{43}$ N. Beni, ${ }^{44}$ S. Czellar, ${ }^{44}$ J. Molnar, ${ }^{44}$ J. Palinkas, ${ }^{44}$ Z. Szillasi, ${ }^{44}$ J. Karancsi, ${ }^{45}$ P. Raics, ${ }^{45}$ Z. L. Trocsanyi, ${ }^{45}$ B. Ujvari,${ }^{45}$ S. K. Swain, ${ }^{46}$ S. B. Beri, ${ }^{47}$ V. Bhatnagar, ${ }^{47}$ N. Dhingra, ${ }^{47}$ R. Gupta, ${ }^{47}$ M. Kaur,${ }^{47}$ M. Z. Mehta, ${ }^{47}$ M. Mittal, ${ }^{47}$ N. Nishu, ${ }^{47}$ A. Sharma,${ }^{47}$ J. B. Singh,${ }^{47}$ A. Kumar, ${ }^{48}$ A. Kumar, ${ }^{48}$ S. Ahuja ${ }^{48}$ A. Bhardwaj, ${ }^{48}$ B. C. Choudhary, ${ }^{48}$ A. Kumar, ${ }^{48}$ S. Malhotra, ${ }^{48}$ M. Naimuddin, ${ }^{48}$ K. Ranjan,${ }^{48}$ V. Sharma, ${ }^{48}$ R. K. Shivpuri ${ }^{48}$ S. Banerjee ${ }^{49}$ S. Bhattacharya ${ }^{49}$ K. Chatterjee, ${ }^{49}$ S. Dutta, ${ }^{49}$ B. Gomber, ${ }^{49}$ S. Jain, ${ }^{49}$ S. Jain, ${ }^{49}$ R. Khurana, ${ }^{49}$ A. Modak,${ }^{49}$ S. Mukherjee ${ }^{49}$ D. Roy, ${ }^{49}$ S. Sarkar ${ }^{49}$ M. Sharan,${ }^{49}$ A. P. Singh ${ }^{49}$ A. Abdulsalam, ${ }^{50}$ D. Dutta, ${ }^{50}$ S. Kailas, ${ }^{50}$ V. Kumar, ${ }^{50}$ A. K. Mohanty, ${ }^{50 c}$ L. M. Pant, ${ }^{50}$ P. Shukla, ${ }^{50}$ A. Topkar, ${ }^{50}$ T. Aziz,${ }^{51}$ R. M. Chatterjee, ${ }^{51}$ S. Ganguly,${ }^{51}$ S. Ghosh, ${ }^{51}$ M. Guchait,${ }^{51 v}$ A. Gurtu, ${ }^{51 w}$ G. Kole, ${ }^{51}$ S. Kumar, ${ }^{51}$ M. Maity ${ }^{51 x}$ G. Majumder, ${ }^{51}$ K. Mazumdar, ${ }^{51}$ G. B. Mohanty ${ }^{51}$ B. Parida, ${ }^{51}$ K. Sudhakar, ${ }^{51}$ N. Wickramage, ${ }^{51 y}$ S. Banerjee, ${ }^{52}$ S. Dugad,${ }^{52}$ H. Arfaei,${ }^{53}$ H. Bakhshiansohi, ${ }^{53}$ H. Behnamian, ${ }^{53}$ S. M. Etesami, ${ }^{53 z}$

A. Fahim, ${ }^{53 a a}$ A. Jafari, ${ }^{53}$ M. Khakzad, ${ }^{53}$ M. Mohammadi Najafabadi, ${ }^{53}$ M. Naseri, ${ }^{53}$ S. Paktinat Mehdiabadi, ${ }^{53}$ B. Safarzadeh, ${ }^{53 b b}$ M. Zeinali, ${ }^{53}$ M. Grunewald, ${ }^{54}$ M. Abbrescia, ${ }^{55 a, 55 b}$ L. Barbone,${ }^{55 a, 55 b}$ C. Calabria, ${ }^{55 a, 55 b}$ S. S. Chhibra, ${ }^{55 a, 55 b}$ A. Colaleo ${ }^{55 a}$ D. Creanza, ${ }^{55 a, 55 c}$ N. De Filippis, ${ }^{55 a, 55 c}$ M. De Palma, ${ }^{55 a, 55 b}$ L. Fiore,${ }^{55 a}$ G. Iaselli, ${ }^{55 a, 55 c}$ G. Maggi, ${ }^{55 a, 55 c}$ M. Maggi, ${ }^{55 a}$ B. Marangelli, ${ }^{55 a, 55 b}$ S. My, ${ }^{55 a, 55 c}$ S. Nuzzo,${ }^{55 a, 55 b}$ N. Pacifico, ${ }^{55 a}$ A. Pompili, ${ }^{55 a, 55 b}$ G. Pugliese,${ }^{55,55 \mathrm{c}}$ R. Radogna,${ }^{55 \mathrm{a}, 55 \mathrm{~b}}$ G. Selvaggi, ${ }^{55 \mathrm{a}, 55 \mathrm{~b}}$ L. Silvestris, ${ }^{55 \mathrm{a}}$ G. Singh,${ }^{55 \mathrm{a}, 55 \mathrm{~b}}$ R. Venditti, ${ }^{55 \mathrm{a}, 55 \mathrm{~b}}$ P. Verwilligen, ${ }^{55 \mathrm{a}}$ G. Zito, ${ }^{55 \mathrm{a}}$ G. Abbiendi, ${ }^{56 \mathrm{a}}$ A. C. Benvenuti, ${ }^{56 \mathrm{a}}$ D. Bonacorsi, ${ }^{56 \mathrm{a}, 56 \mathrm{~b}}$ S. Braibant-Giacomelli, ${ }^{56 \mathrm{a}, 56 \mathrm{~b}}$ L. Brigliadori, ${ }^{56 \mathrm{a}, 56 \mathrm{~b}}$ R. Campanini, ${ }^{56 a, 56 b}$ P. Capiluppi ${ }^{56 a, 56 b}$ A. Castro, ${ }^{56 a, 56 b}$ F. R. Cavallo, ${ }^{56 \mathrm{a}}$ G. Codispoti, ${ }^{56 a, 56 b}$ M. Cuffiani, ${ }^{56 a, 56 b}$ G. M. Dallavalle, ${ }^{56 a}$ F. Fabbri, ${ }^{56 a}$ A. Fanfani, ${ }^{56 a, 56 b}$ D. Fasanella, ${ }^{56 a, 56 b}$ P. Giacomelli ${ }^{56 a}$ C. Grandi, ${ }^{56 a}$ L. Guiducci,${ }^{56 a, 56 b}$ S. Marcellini, ${ }^{56}$ G. Masetti, ${ }^{56 a}$ M. Meneghelli, ${ }^{56,56 b}$ A. Montanari, ${ }^{56 \mathrm{a}}$ F. L. Navarria, ${ }^{56 a, 56 b}$ F. Odorici, ${ }^{56 a}$ A. Perrotta ${ }^{56 a}$ F. Primavera, ${ }^{56 a, 56 b}$ A. M. Rossi, ${ }^{56 a, 56 b}$ T. Rovelli, ${ }^{56 a, 56 b}$ G. P. Siroli,${ }^{56 a, 56 b}$ N. Tosi, ${ }^{56 a, 56 b}$ R. Travaglini, ${ }^{56 a, 56 b}$ S. Albergo, ${ }^{57 a, 57 b}$ G. Cappello, ${ }^{57 \mathrm{a}}$ M. Chiorboli, ${ }^{57,57 \mathrm{~b}}$ S. Costa, ${ }^{57,57 b}$ F. Giordano, ${ }^{57 a, 57 \mathrm{cc}}$ R. Potenza, ${ }^{57 \mathrm{a}, 57 \mathrm{~b}}$ A. Tricomi, ${ }^{57 \mathrm{a}, 57 \mathrm{~b}}$ C. Tuve, ${ }^{57 \mathrm{a}, 57 \mathrm{~b}}$ G. Barbagli, ${ }^{58 \mathrm{a}}$ V. Ciulli, ${ }^{58 \mathrm{a}, 58 \mathrm{~b}}$ C. Civinini, ${ }^{58 \mathrm{a}}$ R. D’Alessandro, ${ }^{58 \mathrm{a}, 58 \mathrm{~b}}$ E. Focardi, ${ }^{58 \mathrm{a}, 58 \mathrm{~b}}$ E. Gallo, ${ }^{58 \mathrm{a}}$ S. Gonzi, ${ }^{58 \mathrm{a}, 58 \mathrm{~b}}$ V. Gori ${ }^{58 a, 58 b}$ P. Lenzi, ${ }^{58 a, 58 b}$ M. Meschini ${ }^{58 \mathrm{a}}$ S. Paoletti, ${ }^{58 \mathrm{a}}$ G. Sguazzoni, ${ }^{58 \mathrm{a}}$ A. Tropiano, ${ }^{58 \mathrm{a}, 58 \mathrm{~b}}$ L. Benussi, ${ }^{59}$ S. Bianco,${ }^{59}$ F. Fabbri, ${ }^{59}$ D. Piccolo, ${ }^{59}$ P. Fabbricatore, ${ }^{60 \mathrm{a}}$ R. Ferretti, ${ }^{60 \mathrm{a}, 60 \mathrm{~b}}$ F. Ferro, ${ }^{60 \mathrm{a}}$ M. Lo Vetere, ${ }^{60 \mathrm{a}, 60 \mathrm{~b}}$ R. Musenich, ${ }^{60 \mathrm{a}}$ E. Robutti, ${ }^{60 \mathrm{a}}$ S. Tosi, ${ }^{60 a, 60 b}$ A. Benaglia, ${ }^{61 a}$ M. E. Dinardo, ${ }^{61 \mathrm{a}, 61 \mathrm{~b}}$ S. Fiorendi, ${ }^{61,61 \mathrm{bc}}$ S. Gennai, ${ }^{61 \mathrm{a}}$ R. Gerosa, ${ }^{61 \mathrm{a}}$ A. Ghezzi, ${ }^{61,61 \mathrm{~b}}$ P. Govoni, ${ }^{61 \mathrm{a}, 61 \mathrm{~b}}$ M. T. Lucchini, ${ }^{61 \mathrm{a}, 61 \mathrm{bc}}$ S. Malvezzi, ${ }^{61 \mathrm{a}}$ R. A. Manzoni, ${ }^{61 \mathrm{a}, 61 \mathrm{bc}}$ A. Martelli, ${ }^{61 \mathrm{a}, 61 \mathrm{bc}}$ B. Marzocchi, ${ }^{61 \mathrm{a}}$ D. Menasce, ${ }^{61 \mathrm{a}}$ L. Moroni, ${ }^{61 \mathrm{a}}$ M. Paganoni, ${ }^{61 \mathrm{a}, 61 \mathrm{~b}}$ D. Pedrini, ${ }^{61 \mathrm{a}}$ S. Ragazzi, ${ }^{61 \mathrm{a}, 61 \mathrm{~b}}$ N. Redaelli, ${ }^{61 \mathrm{a}}$ T. Tabarelli de Fatis, ${ }^{61 \mathrm{a}, 61 \mathrm{~b}}$ S. Buontempo, ${ }^{62 \mathrm{a}}$ N. Cavallo, ${ }^{62 a, 62 \mathrm{c}}$ F. Fabozzi, ${ }^{62 a, 62 \mathrm{c}}$ A. O. M. Iorio, ${ }^{62 a, 62 \mathrm{~b}}$ L. Lista, ${ }^{62 \mathrm{a}}$ S. Meola ${ }^{62 \mathrm{a}, 62 \mathrm{dc}}$ M. Merola, ${ }^{62 \mathrm{a}}$ P. Paolucci, ${ }^{62 a c}$ P. Azzi, ${ }^{63 a}$ N. Bacchetta, ${ }^{63 a}$ D. Bisello, ${ }^{63 a, 63 b}$ A. Branca, ${ }^{63 a, 63 b}$ R. Carlin, ${ }^{63 a, 63 b}$ P. Checchia, ${ }^{63 a}$ T. Dorigo, ${ }^{63 a}$ M. Galanti, ${ }^{63 a, 63 b c}$ F. Gasparini, ${ }^{63 a, 63 b}$ U. Gasparini, ${ }^{63 a, 63 b}$ P. Giubilato, ${ }^{63 a, 63 b}$ A. Gozzelino, ${ }^{63 a}$ K. Kanishchev, ${ }^{63,63 c}$ S. Lacaprara, ${ }^{63 a}$ I. Lazzizzera, ${ }^{63 a, 63 c}$ M. Margoni, ${ }^{63 a, 63 b}$ A. T. Meneguzzo, ${ }^{63 a, 63 b}$ M. Passaseo, ${ }^{63 a}$ J. Pazzini, ${ }^{63 a, 63 b}$ M. Pegoraro, ${ }^{63 a}$ N. Pozzobon, ${ }^{63 a, 63 b}$ P. Ronchese,${ }^{63 a, 63 b}$ F. Simonetto, ${ }^{63 a, 63 b}$ E. Torassa, ${ }^{63 a}$ M. Tosi,${ }^{63 a, 63 b}$ A. Triossi, ${ }^{63 a}$ S. Ventura, ${ }^{63 a}$ A. Zucchetta, ${ }^{63 a, 63 b}$ G. Zumerle ${ }^{63 a, 63 b}$ M. Gabusi, ${ }^{64 a, 64 b}$ S. P. Ratti ${ }^{64 a, 64 b}$ C. Riccardi, ${ }^{64 a, 64 b}$ P. Vitulo, ${ }^{64 a, 64 b}$ M. Biasini, ${ }^{65 a, 65 b}$ G. M. Bilei, ${ }^{65 a}$ L. Fanò, ${ }^{65 a, 65 b}$ P. Lariccia,${ }^{65 a, 65 b}$ G. Mantovani ${ }^{65 a, 65 b}$ M. Menichelli, ${ }^{65 a}$ F. Romeo, ${ }^{65 a, 65 b}$ A. Saha ${ }^{65 a}$ A. Santocchia, ${ }^{65 a, 65 b}$ A. Spiezia, ${ }^{65 a, 65 b}$ K. Androsov ${ }^{66 a c c}$ P. Azzurri, ${ }^{66 a}$ G. Bagliesi, ${ }^{66 a}$ J. Bernardini, ${ }^{66 a}$ T. Boccali, ${ }^{66 a}$ G. Broccolo, ${ }^{66 a, 66 c}$ R. Castaldi, ${ }^{66 a}$ M. A. Ciocci, ${ }^{66 a c c}$ R. Dell'Orso, ${ }^{66 a}$ F. Fiori, ${ }^{66 a, 66 c}$ L. Foà ${ }^{66 a, 66 c}$ A. Giassi, ${ }^{66 a}$ M. T. Grippo, ${ }^{66 a c c}$ A. Kraan, ${ }^{66 a}$ F. Ligabue, ${ }^{66 a, 66 c}$ T. Lomtadze, ${ }^{66 \mathrm{a}}$ L. Martini, ${ }^{66 a, 66 \mathrm{~b}}$ A. Messineo, ${ }^{66 \mathrm{a}, 66 \mathrm{~b}}$ C. S. Moon, ${ }^{66 a d d}$ F. Palla, ${ }^{66 a}$ A. Rizzi, ${ }^{66 a, 66 b}$ A. Savoy-Navarro, ${ }^{66 a e e}$ A. T. Serban, ${ }^{66 a}$ P. Spagnolo, ${ }^{66 a}$ P. Squillacioti, ${ }^{66 a c c}$ R. Tenchini, ${ }^{66 a}$ G. Tonelli, ${ }^{66 a, 66 b}$ A. Venturi, ${ }^{66 a}$ P. G. Verdini, ${ }^{66 a}$ C. Vernieri, ${ }^{66 a, 66 c}$ L. Barone, ${ }^{67 a, 67 b}$ F. Cavallari, ${ }^{67 a}$ D. Del Re, ${ }^{67 a, 67 b}$

M. Diemoz, ${ }^{67 a}$ M. Grassi, ${ }^{67 a, 67 b}$ C. Jorda, ${ }^{67 a}$ E. Longo, ${ }^{67 a, 67 b}$ F. Margaroli,${ }^{67 a, 67 b}$ P. Meridiani,${ }^{67 a}$ F. Micheli, ${ }^{67 a, 67 b}$ S. Nourbakhsh, ${ }^{67 a, 67 b}$ G. Organtini, ${ }^{67 a, 67 b}$ R. Paramatti, ${ }^{67 a}$ S. Rahatlou, ${ }^{67 a, 67 b}$ C. Rovelli, ${ }^{67 a}$ L. Soffi, ${ }^{67 a, 67 b}$ P. Traczyk, ${ }^{67 a, 67 b}$ N. Amapane, ${ }^{68 a, 68 b}$ R. Arcidiacono, ${ }^{68 a, 68 c}$ S. Argiro, ${ }^{68 a, 68 b}$ M. Arneodo, ${ }^{68 a, 68 c}$ R. Bellan, ${ }^{68 a, 68 b}$ C. Biino, ${ }^{68 a}$ N. Cartiglia, ${ }^{68 a}$ S. Casasso, ${ }^{68 a, 68 b}$ M. Costa, ${ }^{68 a, 68 b}$ A. Degano, ${ }^{68 a, 68 b}$ N. Demaria ${ }^{68 a}$ C. Mariotti, ${ }^{68 a}$ S. Maselli, ${ }^{68 a}$ E. Migliore, ${ }^{68 a, 68 b}$ V. Monaco, ${ }^{68 a, 68 b}$ M. Musich, ${ }^{68 a}$ M. M. Obertino, ${ }^{68 a, 68 c}$ G. Ortona, ${ }^{68 a, 68 b}$ L. Pacher,${ }^{68 a, 68 b}$ N. Pastrone, ${ }^{68 a}$ M. Pelliccioni ${ }^{68 a c}$ A. Potenza ${ }^{68 a, 68 b}$ A. Romero, ${ }^{68 a, 68 b}$ M. Ruspa, ${ }^{68 a, 68 c}$ R. Sacchi, ${ }^{68 a, 68 b}$ A. Solano, ${ }^{68 a, 68 b}$ A. Staiano, ${ }^{68 a}$ U. Tamponi, ${ }^{68 a}$ S. Belforte ${ }^{69 a}$ V. Candelise,${ }^{69 a, 69 b}$ M. Casarsa ${ }^{69 a}$ F. Cossutti, ${ }^{69 a}$ G. Della Ricca ${ }^{69 a, 69 b}$ B. Gobbo, ${ }^{69 a}$ C. La Licata, ${ }^{69 a, 69 b}$ M. Marone ${ }^{69 a, 69 b}$ D. Montanino, ${ }^{69 a, 69 b}$ A. Penzo, ${ }^{69 a}$ A. Schizzi, ${ }^{69 a, 69 b}$ T. Umer, ${ }^{69 a, 69 b}$ A. Zanetti, ${ }^{69 a}$ S. Chang, ${ }^{70}$ T. Y. Kim, ${ }^{70}$ S. K. Nam, ${ }^{70}$ D. H. Kim, ${ }^{71}$ G. N. Kim, ${ }^{71}$ J. E. Kim, ${ }^{71}$ M. S. Kim,${ }^{71}$ D. J. Kong, ${ }^{71}$ S. Lee, ${ }^{71}$ Y. D. Oh, ${ }^{71}$ H. Park, ${ }^{71}$ D. C. Son, ${ }^{71}$ J. Y. Kim, ${ }^{72}$ Z. J. Kim, ${ }^{72}$ S. Song, ${ }^{72}$ S. Choi, ${ }^{73}$ D. Gyun, ${ }^{73}$ B. Hong, ${ }^{73}$ M. Jo, ${ }^{73}$ H. Kim, ${ }^{73}$ Y. Kim, ${ }^{73}$ K. S. Lee, ${ }^{73}$ S. K. Park, ${ }^{73}$ 
Y. Roh, ${ }^{73}$ M. Choi,${ }^{74}$ J. H. Kim, ${ }^{74}$ C. Park, ${ }^{74}$ I. C. Park ${ }^{74}$ S. Park, ${ }^{74}$ G. Ryu, ${ }^{74}$ Y. Choi,${ }^{75}$ Y. K. Choi, ${ }^{75}$ J. Goh,${ }^{75}$ E. Kwon, ${ }^{75}$ B. Lee, ${ }^{75}$ J. Lee ${ }^{75}$ S. Lee ${ }^{75}$ H. Seo, ${ }^{75}$ I. Yu, ${ }^{75}$ A. Juodagalvis, ${ }^{76}$ J. R. Komaragiri, ${ }^{77}$ H. Castilla-Valdez, ${ }^{78}$ E. De La CruzBurelo, ${ }^{78}$ I. Heredia-de La Cruz, ${ }^{78 f f}$ R. Lopez-Fernandez, ${ }^{78}$ J. Martínez-Ortega, ${ }^{78}$ A. Sanchez-Hernandez ${ }^{78}$

L. M. Villasenor-Cendejas, ${ }^{78}$ S. Carrillo Moreno, ${ }^{79}$ F. Vazquez Valencia,${ }^{79}$ H. A. Salazar Ibarguen, ${ }^{80}$ E. Casimiro Linares ${ }^{81}$ A. Morelos Pineda, ${ }^{81}$ D. Krofcheck, ${ }^{82}$ P. H. Butler, ${ }^{83}$ R. Doesburg, ${ }^{83}$ S. Reucroft,${ }^{83}$ M. Ahmad, ${ }^{84}$ M. I. Asghar,${ }^{84}$ J. Butt,${ }^{84}$ H. R. Hoorani, ${ }^{84}$ S. Khalid ${ }^{84}$ W. A. Khan,${ }^{84}$ T. Khurshid, ${ }^{84}$ S. Qazi,${ }^{84}$ M. A. Shah, ${ }^{84}$ M. Shoaib ${ }^{84}$ H. Bialkowska ${ }^{85}$ M. Bluj, ${ }^{85 g}$ B. Boimska, ${ }^{85}$ T. Frueboes, ${ }^{85}$ M. Górski, ${ }^{85}$ M. Kazana ${ }^{85}$ K. Nawrocki, ${ }^{85}$ K. Romanowska-Rybinska ${ }^{85}$

M. Szleper, ${ }^{85}$ G. Wrochna,${ }^{85}$ P. Zalewski,${ }^{85}$ G. Brona,${ }^{86}$ K. Bunkowski, ${ }^{86}$ M. Cwiok, ${ }^{86}$ W. Dominik, ${ }^{86}$ K. Doroba, ${ }^{86}$ A. Kalinowski, ${ }^{86}$ M. Konecki, ${ }^{86}$ J. Krolikowski, ${ }^{86}$ M. Misiura ${ }^{86}$ W. Wolszczak, ${ }^{86}$ P. Bargassa, ${ }^{87}$ C. Beirão Da Cruz E Silva, ${ }^{87}$ P. Faccioli, ${ }^{87}$ P. G. Ferreira Parracho,${ }^{87}$ M. Gallinaro, ${ }^{87}$ F. Nguyen, ${ }^{87}$ J. Rodrigues Antunes, ${ }^{87}$ J. Seixas, ${ }^{87 c}$ J. Varela, ${ }^{87}$ P. Vischia, ${ }^{87}$ M. Gavrilenko, ${ }^{88}$ I. Golutvin, ${ }^{88}$ I. Gorbunov, ${ }^{88}$ A. Kamenev, ${ }^{88}$ V. Karjavin, ${ }^{88}$ V. Konoplyanikov, ${ }^{88}$ G. Kozlov, ${ }^{88}$ A. Lanev, ${ }^{88}$ A. Malakhov, ${ }^{88}$ V. Matveev, ${ }^{88 h h}$ P. Moisenz ${ }^{88}$ V. Palichik ${ }^{88}$ V. Perelygin, ${ }^{88}$ M. Savina, ${ }^{88}$ S. Shmatov ${ }^{88}$ N. Skatchkov, ${ }^{88}$ V. Smirnov, ${ }^{88}$ A. Zarubin ${ }^{88}$ V. Golovtsov, ${ }^{89}$ Y. Ivanov, ${ }^{89}$ V. Kim, ${ }^{89}$ P. Levchenko, ${ }^{89}$ V. Murzin,${ }^{89}$

V. Oreshkin, ${ }^{89}$ I. Smirnov, ${ }^{89}$ V. Sulimov, ${ }^{89}$ L. Uvarov ${ }^{89}$ S. Vavilov, ${ }^{89}$ A. Vorobyev, ${ }^{89}$ A. Vorobyev, ${ }^{89}$ Y. Andreev, ${ }^{90}$ A. Dermenev, ${ }^{90}$ S. Gninenko, ${ }^{90}$ N. Golubev, ${ }^{90}$ M. Kirsanov, ${ }^{90}$ N. Krasnikov, ${ }^{90}$ A. Pashenkov, ${ }^{90}$ D. Tlisov, ${ }^{90}$ A. Toropin,,${ }^{90}$ V. Epshteyn, ${ }^{91}$ V. Gavrilov, ${ }^{91}$ N. Lychkovskaya,${ }^{91}$ V. Popov,${ }^{91}$ G. Safronov, ${ }^{91}$ S. Semenov, ${ }^{91}$ A. Spiridonov, ${ }^{91}$ V. Stolin, ${ }^{91}$ E. Vlasov, ${ }^{91}$ A. Zhokin, ${ }^{91}$ V. Andreev, ${ }^{92}$ M. Azarkin, ${ }^{92}$ I. Dremin, ${ }^{92}$ M. Kirakosyan, ${ }^{92}$ A. Leonidov, ${ }^{92}$ G. Mesyats, ${ }^{92}$ S. V. Rusakov, ${ }^{92}$ A. Vinogradov, ${ }^{92}$ A. Belyaev, ${ }^{93}$ E. Boos,${ }^{93}$ V. Bunichev,${ }^{93}$ M. Dubinin, ${ }^{93 \mathrm{~h}}$ L. Dudko, ${ }^{93}$ A. Ershov, ${ }^{93}$ A. Gribushin, ${ }^{93}$ V. Klyukhin, ${ }^{93}$ O. Kodolova, ${ }^{93}$ I. Lokhtin, ${ }^{93}$ S. Obraztsov, ${ }^{93}$ S. Petrushanko, ${ }^{93}$ V. Savrin, ${ }^{93}$ I. Azhgirey,${ }^{94}$ I. Bayshev,${ }^{94}$ S. Bitioukov, ${ }^{94}$ V. Kachanov,${ }^{94}$ A. Kalinin, ${ }^{94}$ D. Konstantinov,${ }^{94}$ V. Krychkine, ${ }^{94}$ V. Petrov, ${ }^{94}$ R. Ryutin, ${ }^{94}$ A. Sobol, ${ }^{94}$ L. Tourtchanovitch, ${ }^{94}$ S. Troshin, ${ }^{94}$ N. Tyurin, ${ }^{94}$ A. Uzunian, ${ }^{94}$ A. Volkov, ${ }^{94}$ P. Adzic, ${ }^{95 i i}$ M. Djordjevic,${ }^{95}$ M. Ekmedzic, ${ }^{95}$ J. Milosevic, ${ }^{95}$ M. Aguilar-Benitez, ${ }^{96}$ J. Alcaraz Maestre, ${ }^{96}$ C. Battilana, ${ }^{96}$ E. Calvo, ${ }^{96}$ M. Cerrada,${ }^{96}$ M. Chamizo Llatas, ${ }^{96 c}$ N. Colino, ${ }^{96}$ B. De La Cruz,${ }^{96}$ A. Delgado Peris, ${ }^{96}$ D. Domínguez Vázquez, ${ }^{96}$ C. Fernandez Bedoya,${ }^{96}$ J. P. Fernández Ramos, ${ }^{96}$ A. Ferrando,${ }^{96}$ J. Flix, ${ }^{96}$ M. C. Fouz,${ }^{96}$ P. Garcia-Abia, ${ }^{96}$ O. Gonzalez Lopez, ${ }^{96}$ S. Goy Lopez,${ }^{96}$ J. M. Hernandez,${ }^{96}$ M. I. Josa, ${ }^{96}$ G. Merino, ${ }^{96}$ E. Navarro De Martino,,${ }^{96}$ J. Puerta Pelayo, ${ }^{96}$ A. Quintario Olmeda,${ }^{96}$ I. Redondo, ${ }^{96}$ L. Romero, ${ }^{96}$ M. S. Soares,${ }^{96}$ C. Willmott,${ }^{96}$ C. Albajar, ${ }^{97}$ J. F. de Trocóniz, ${ }^{97}$ M. Missiroli ${ }^{97}$ H. Brun, ${ }^{98}$ J. Cuevas, ${ }^{98}$ J. Fernandez Menendez, ${ }^{98}$ S. Folgueras, ${ }^{98}$ I. Gonzalez Caballero, ${ }^{98}$ L. Lloret Iglesias, ${ }^{98}$ J. A. Brochero Cifuentes, ${ }^{99}$ I. J. Cabrillo, ${ }^{99}$ A. Calderon, ${ }^{99}$ S. H. Chuang, ${ }^{99}$ J. Duarte Campderros, ${ }^{99}$ M. Fernandez, ${ }^{99}$ G. Gomez, ${ }^{99}$ J. Gonzalez Sanchez, ${ }^{99}$ A. Graziano, ${ }^{99}$ A. Lopez Virto, ${ }^{99}$ J. Marco,${ }^{99}$ R. Marco,${ }^{99}$ C. Martinez Rivero, ${ }^{99}$ F. Matorras,${ }^{99}$ F. J. Munoz Sanchez, ${ }^{99}$ J. Piedra Gomez, ${ }^{99}$ T. Rodrigo, ${ }^{99}$ A. Y. Rodríguez-Marrero, ${ }^{99}$ A. Ruiz-Jimeno, ${ }^{99}$ L. Scodellaro,${ }^{99}$ I. Vila, ${ }^{99}$ R. Vilar Cortabitarte, ${ }^{99}$ D. Abbaneo, ${ }^{100}$ E. Auffray, ${ }^{100}$ G. Auzinger, ${ }^{100}$ M. Bachtis, ${ }^{100}$ P. Baillon, ${ }^{100}$ A. H. Ball, ${ }^{100}$ D. Barney, ${ }^{100}$ J. Bendavid, ${ }^{100}$ L. Benhabib, ${ }^{100}$ J. F. Benitez, ${ }^{100}$ C. Bernet, ${ }^{100 i}$ G. Bianchi, ${ }^{100}$ P. Bloch, ${ }^{100}$ A. Bocci, ${ }^{100}$ A. Bonato, ${ }^{100}$ O. Bondu, ${ }^{100}$ C. Botta, ${ }^{100}$ H. Breuker, ${ }^{100}$ T. Camporesi, ${ }^{100}$ G. Cerminara, ${ }^{100}$ T. Christiansen, ${ }^{100}$ J. A. Coarasa Perez ${ }^{100}$ S. Colafranceschi, ${ }^{100 j \mathrm{jj}}$ M. D’Alfonso, ${ }^{100}$ D. d'Enterria, ${ }^{100}$ A. Dabrowski, ${ }^{100}$ A. David, ${ }^{100}$ F. De Guio, ${ }^{100}$ A. De Roeck, ${ }^{100}$ S. De Visscher ${ }^{100}$ S. Di Guida, ${ }^{100}$ M. Dobson,${ }^{100}$ N. Dupont-Sagorin, ${ }^{100}$ A. Elliott-Peisert ${ }^{100}$ J. Eugster,${ }^{100}$ G. Franzoni, ${ }^{100}$ W. Funk,${ }^{100}$ M. Giffels, ${ }^{100}$ D. Gigi, ${ }^{100}$ K. Gill, ${ }^{100}$ M. Girone,${ }^{100}$ M. Giunta, ${ }^{100}$ F. Glege, ${ }^{100}$ R. Gomez-Reino Garrido, ${ }^{100}$ S. Gowdy, ${ }^{100}$ R. Guida, ${ }^{100}$ J. Hammer, ${ }^{100}$ M. Hansen, ${ }^{100}$ P. Harris, ${ }^{100}$ V. Innocente, ${ }^{100}$ P. Janot, ${ }^{100}$ E. Karavakis, ${ }^{100}$ K. Kousouris,${ }^{100}$ K. Krajczar, ${ }^{100}$ P. Lecoq ${ }^{100}$ C. Lourenço, ${ }^{100}$ N. Magini, ${ }^{100}$ L. Malgeri, ${ }^{100}$ M. Mannelli, ${ }^{100}$ L. Masetti, ${ }^{100}$ F. Meijers ${ }^{100}$ S. Mersi, ${ }^{100}$ E. Meschi ${ }^{100}$ F. Moortgat ${ }^{100}$ M. Mulders,${ }^{100}$ P. Musella, ${ }^{100}$ L. Orsini, ${ }^{100}$ E. Palencia Cortezon, ${ }^{100}$ E. Perez,${ }^{100}$ L. Perrozzi, ${ }^{100}$ A. Petrilli, ${ }^{100}$ G. Petrucciani, ${ }^{100}$ A. Pfeiffer,${ }^{100}$ M. Pierini, ${ }^{100}$ M. Pimiä, ${ }^{100}$ D. Piparo, ${ }^{100}$ M. Plagge, ${ }^{100}$ A. Racz, ${ }^{100}$ W. Reece, ${ }^{100}$ G. Rolandi, ${ }^{100 k}$ M. Rovere, ${ }^{100}$ H. Sakulin, ${ }^{100}$ F. Santanastasio, ${ }^{100}$ C. Schäfer, ${ }^{100}$ C. Schwick, ${ }^{100}$ S. Sekmen, ${ }^{100}$ A. Sharma, ${ }^{100}$ P. Siegrist, ${ }^{100}$ P. Silva, ${ }^{100}$ M. Simon, ${ }^{100}$ P. Sphicas, ${ }^{10011}$ J. Steggemann, ${ }^{100}$ B. Stieger, ${ }^{100}$ M. Stoye, ${ }^{100}$ A. Tsirou, ${ }^{100}$ G. I. Veres,${ }^{100 u}$ J. R. Vlimant, ${ }^{100}$ H. K. Wöhri, ${ }^{100}$ W. D. Zeuner,${ }^{100}$ W. Bertl, ${ }^{101}$ K. Deiters, ${ }^{101}$ W. Erdmann, ${ }^{101}$ R. Horisberger,${ }^{101}$ Q. Ingram, ${ }^{101}$ H. C. Kaestli, ${ }^{101}$ S. König, ${ }^{101}$ D. Kotlinski, ${ }^{101}$ U. Langenegger, ${ }^{101}$ D. Renker, ${ }^{101}$ T. Rohe, ${ }^{101}$ F. Bachmair, ${ }^{102}$ L. Bäni, ${ }^{102}$ L. Bianchini, ${ }^{102}$ P. Bortignon, ${ }^{102}$ M. A. Buchmann, ${ }^{102}$ B. Casal, ${ }^{102}$ N. Chanon, ${ }^{102}$ A. Deisher, ${ }^{102}$ G. Dissertori, ${ }^{102}$ M. Dittmar, ${ }^{102}$ M. Donegà, ${ }^{102}$ M. Dünser, ${ }^{102}$ P. Eller, ${ }^{102}$ C. Grab, ${ }^{102}$ D. Hits, ${ }^{102}$ W. Lustermann, ${ }^{102}$ B. Mangano, ${ }^{102}$ A. C. Marini, ${ }^{102}$ P. Martinez Ruiz del Arbol, ${ }^{102}$ D. Meister, ${ }^{102}$ N. Mohr, ${ }^{102}$ C. Nägeli, ${ }^{102 \mathrm{~mm}}$ P. Nef, ${ }^{102}$ F. Nessi-Tedaldi, ${ }^{102}$ F. Pandolfi,,${ }^{102}$ L. Pape,${ }^{102}$ F. Pauss, ${ }^{102}$ M. Peruzzi, ${ }^{102}$ M. Quittnat, ${ }^{102}$ F. J. Ronga, ${ }^{102}$ M. Rossini, ${ }^{102}$ A. Starodumov, ${ }^{102 n n}$ M. Takahashi, ${ }^{102}$ L. Tauscher, ${ }^{102 a}$ 
K. Theofilatos, ${ }^{102}$ D. Treille, ${ }^{102}$ R. Wallny, ${ }^{102}$ H. A. Weber, ${ }^{102}$ C. Amsler, ${ }^{10300}$ V. Chiochia, ${ }^{103}$ A. De Cosa,${ }^{103}$ C. Favaro, ${ }^{103}$ A. Hinzmann, ${ }^{103}$ T. Hreus, ${ }^{103}$ M. Ivova Rikova, ${ }^{103}$ B. Kilminster, ${ }^{103}$ B. Millan Mejias, ${ }^{103}$ J. Ngadiuba, ${ }^{103}$ P. Robmann, ${ }^{103}$ H. Snoek, ${ }^{103}$ S. Taroni, ${ }^{103}$ M. Verzetti, ${ }^{103}$ Y. Yang, ${ }^{103}$ M. Cardaci, ${ }^{104}$ K. H. Chen, ${ }^{104}$ C. Ferro, ${ }^{104}$ C. M. Kuo, ${ }^{104}$ S. W. Li, ${ }^{104}$ W. Lin, ${ }^{104}$ Y. J. Lu, ${ }^{104}$ R. Volpe, ${ }^{104}$ S. S. Yu, ${ }^{104}$ P. Bartalini, ${ }^{105}$ P. Chang, ${ }^{105}$ Y. H. Chang, ${ }^{105}$ Y. W. Chang, ${ }^{105}$ Y. Chao, ${ }^{105}$ K. F. Chen, ${ }^{105}$ P. H. Chen, ${ }^{105}$ C. Dietz, ${ }^{105}$ U. Grundler, ${ }^{105}$ W.-S. Hou, ${ }^{105}$ Y. Hsiung, ${ }^{105}$ K. Y. Kao, ${ }^{105}$ Y. J. Lei, ${ }^{105}$ Y. F. Liu, ${ }^{105}$ R.-S. Lu, ${ }^{105}$ D. Majumder, ${ }^{105}$ E. Petrakou, ${ }^{105}$ X. Shi, ${ }^{105}$ J. G. Shiu, ${ }^{105}$ Y. M. Tzeng, ${ }^{105}$ M. Wang, ${ }^{105}$ R. Wilken, ${ }^{105}$ B. Asavapibhop, ${ }^{106}$ N. Suwonjandee, ${ }^{106}$ A. Adiguzel, ${ }^{107}$ M. N. Bakirci, ${ }^{107 p p}$ S. Cerci, ${ }^{107 q q}$ C. Dozen, ${ }^{107}$ I. Dumanoglu, ${ }^{107}$ E. Eskut, ${ }^{107}$ S. Girgis, ${ }^{107}$ G. Gokbulut, ${ }^{107}$ E. Gurpinar, ${ }^{107}$ I. Hos, ${ }^{107}$ E. E. Kangal, ${ }^{107}$ A. Kayis Topaksu, ${ }^{107}$ G. Onengut, ${ }^{107 r r}$ K. Ozdemir, ${ }^{107}$ S. Ozturk, ${ }^{107 p p}$ A. Polatoz, ${ }^{107}$ K. Sogut, ${ }^{107 s s}$ D. Sunar Cerci, ${ }^{107 q q}$ B. Tali, ${ }^{107 q q}$ H. Topakli, ${ }^{107 p p}$ M. Vergili, ${ }^{107}$ I. V. Akin, ${ }^{108}$ T. Aliev, ${ }^{108}$ B. Bilin, ${ }^{108}$ S. Bilmis, ${ }^{108}$ M. Deniz, ${ }^{108}$ H. Gamsizkan, ${ }^{108}$ A. M. Guler, ${ }^{108}$ G. Karapinar, ${ }^{108 t t}$ K. Ocalan, ${ }^{108}$ A. Ozpineci, ${ }^{108}$ M. Serin, ${ }^{108}$ R. Sever, ${ }^{108}$ U. E. Surat, ${ }^{108}$ M. Yalvac, ${ }^{108}$ M. Zeyrek, ${ }^{108}$ E. Gülmez, ${ }^{109}$ B. Isildak, ${ }^{109 u \mathrm{u}}$ M. Kaya, ${ }^{109 \mathrm{vv}}$ O. Kaya ${ }^{109 \mathrm{vv}}$ S. Ozkorucuklu ${ }^{109 \mathrm{ww}}$ H. Bahtiyar, ${ }^{110 \mathrm{xx}}$ E. Barlas, ${ }^{110}$ K. Cankocak, ${ }^{110}$ Y. O. Günaydin, ${ }^{10 y y}$ F. I. Vardarl,,${ }^{110}$ M. Yücel,${ }^{110}$ L. Levchuk, ${ }^{111}$ P. Sorokin,,${ }^{11}$ J. J. Brooke, ${ }^{112}$ E. Clement, ${ }^{112}$ D. Cussans, ${ }^{112}$ H. Flacher, ${ }^{112}$ R. Frazier, ${ }^{112}$ J. Goldstein, ${ }^{112}$ M. Grimes, ${ }^{112}$ G. P. Heath, ${ }^{112}$ H. F. Heath, ${ }^{112}$ J. Jacob, ${ }^{112}$ L. Kreczko, ${ }^{112}$ C. Lucas, ${ }^{112}$ Z. Meng, ${ }^{112}$ D. M. Newbold, ${ }^{112 z z}$ S. Paramesvaran, ${ }^{112}$ A. Poll, ${ }^{112}$ S. Senkin, ${ }^{112}$ V. J. Smith, ${ }^{112}$ T. Williams, ${ }^{112}$ K. W. Bell, ${ }^{113}$ A. Belyaev, ${ }^{113 a a a}$ C. Brew, ${ }^{113}$ R. M. Brown, ${ }^{113}$ D. J. A. Cockerill, ${ }^{113}$ J. A. Coughlan, ${ }^{113}$ K. Harder, ${ }^{113}$ S. Harper, ${ }^{113}$ J. Ilic, ${ }^{113}$ E. Olaiya, ${ }^{113}$ D. Petyt, ${ }^{113}$ C. H. Shepherd-Themistocleous, ${ }^{113}$ A. Thea, ${ }^{113}$ I. R. Tomalin, ${ }^{113}$ W. J. Womersley, ${ }^{113}$ S. D. Worm, ${ }^{113}$ M. Baber, ${ }^{114}$ R. Bainbridge, ${ }^{114}$ O. Buchmuller, ${ }^{114}$ D. Burton, ${ }^{114}$ D. Colling, ${ }^{114}$ N. Cripps, ${ }^{114}$ M. Cutajar, ${ }^{114}$ P. Dauncey, ${ }^{114}$ G. Davies, ${ }^{114}$ M. Della Negra, ${ }^{114}$ W. Ferguson, ${ }^{114}$ J. Fulcher, ${ }^{114}$ D. Futyan, ${ }^{114}$ A. Gilbert, ${ }^{114}$ A. Guneratne Bryer, ${ }^{114}$ G. Hall, ${ }^{114}$ Z. Hatherell, ${ }^{114}$ J. Hays, ${ }^{114}$ G. Iles, ${ }^{114}$ M. Jarvis, ${ }^{114}$ G. Karapostoli, ${ }^{114}$ M. Kenzie, ${ }^{114}$ R. Lane, ${ }^{114}$ R. Lucas, ${ }^{114 z z}$ L. Lyons, ${ }^{114}$ A.-M. Magnan, ${ }^{114}$ J. Marrouche, ${ }^{114}$ B. Mathias, ${ }^{114}$ R. Nandi, ${ }^{114}$ J. Nash, ${ }^{114}$ A. Nikitenko, ${ }^{114 n n}$ J. Pela, ${ }^{114}$ M. Pesaresi, ${ }^{114}$ K. Petridis, ${ }^{114}$ M. Pioppi,${ }^{114 b b b}$ D. M. Raymond, ${ }^{114}$ S. Rogerson, ${ }^{114}$ A. Rose, ${ }^{114}$ C. Seez, ${ }^{114}$ P. Sharp ${ }^{114 a}$ A. Sparrow, ${ }^{114}$ A. Tapper, ${ }^{114}$ M. Vazquez Acosta, ${ }^{114}$ T. Virdee,${ }^{114}$ S. Wakefield ${ }^{114}$ N. Wardle, ${ }^{114}$ J. E. Cole,${ }^{115}$ P. R. Hobson, ${ }^{115}$ A. Khan, ${ }^{115}$ P. Kyberd, ${ }^{115}$ D. Leggat, ${ }^{115}$ D. Leslie, ${ }^{115}$ W. Martin, ${ }^{115}$ I. D. Reid, ${ }^{115}$ P. Symonds, ${ }^{115}$ L. Teodorescu, ${ }^{115}$ M. Turner, ${ }^{115}$ J. Dittmann, ${ }^{116}$ K. Hatakeyama, ${ }^{116}$ A. Kasmi, ${ }^{116}$ H. Liu, ${ }^{116}$ T. Scarborough, ${ }^{116}$ O. Charaf, ${ }^{117}$ S. I. Cooper, ${ }^{117}$ C. Henderson, ${ }^{117}$ P. Rumerio, ${ }^{117}$ A. Avetisyan, ${ }^{118}$ T. Bose, ${ }^{118}$ C. Fantasia, ${ }^{118}$ A. Heister, ${ }^{118}$ P. Lawson, ${ }^{118}$ D. Lazic, ${ }^{118}$ J. Rohlf, ${ }^{118}$ D. Sperka, ${ }^{118}$ J. St. John, ${ }^{118}$ L. Sulak, ${ }^{118}$ J. Alimena, ${ }^{119}$ S. Bhattacharya, ${ }^{119}$ G. Christopher, ${ }^{119}$ D. Cutts,${ }^{119}$ Z. Demiragli, ${ }^{119}$ A. Ferapontov, ${ }^{119}$ A. Garabedian ${ }^{119}$ U. Heintz, ${ }^{119}$ S. Jabeen, ${ }^{119}$ G. Kukartsev, ${ }^{119}$ E. Laird ${ }^{119}$ G. Landsberg, ${ }^{119}$ M. Luk, ${ }^{119}$ M. Narain, ${ }^{119}$ M. Segala, ${ }^{119}$ T. Sinthuprasith, ${ }^{119}$ T. Speer, ${ }^{119}$ J. Swanson, ${ }^{119}$ R. Breedon, ${ }^{120}$ G. Breto, ${ }^{120}$ M. Calderon De La Barca Sanchez, ${ }^{120}$ S. Chauhan, ${ }^{120}$ M. Chertok, ${ }^{120}$ J. Conway, ${ }^{120}$ R. Conway, ${ }^{120}$ P. T. Cox,${ }^{120}$ R. Erbacher, ${ }^{120}$ M. Gardner, ${ }^{120}$ W. Ko, ${ }^{120}$ A. Kopecky, ${ }^{120}$ R. Lander, ${ }^{120}$ T. Miceli, ${ }^{120}$ D. Pellett, ${ }^{120}$ J. Pilot, ${ }^{120}$ F. Ricci-Tam, ${ }^{120}$ B. Rutherford, ${ }^{120}$ M. Searle, ${ }^{120}$ S. Shalhout, ${ }^{120}$ J. Smith, ${ }^{120}$ M. Squires ${ }^{120}$ M. Tripathi, ${ }^{120}$ S. Wilbur, ${ }^{120}$ R. Yohay, ${ }^{120}$ V. Andreev, ${ }^{121}$ D. Cline, ${ }^{121}$ R. Cousins, ${ }^{121}$ S. Erhan, ${ }^{121}$ P. Everaerts, ${ }^{121}$ C. Farrell, ${ }^{121}$ M. Felcini, ${ }^{121}$ J. Hauser, ${ }^{121}$ M. Ignatenko, ${ }^{121}$ C. Jarvis, ${ }^{121}$ G. Rakness, ${ }^{121}$ P. Schlein, ${ }^{121 a}$ E. Takasugi, ${ }^{121}$ V. Valuev, ${ }^{121}$ M. Weber, ${ }^{121}$ J. Babb, ${ }^{122}$ R. Clare, ${ }^{122}$ J. Ellison, ${ }^{122}$ J. W. Gary, ${ }^{122}$ G. Hanson, ${ }^{122}$ J. Heilman, ${ }^{122}$ P. Jandir, ${ }^{122}$ F. Lacroix, ${ }^{122}$ H. Liu, ${ }^{122}$ O. R. Long, ${ }^{122}$ A. Luthra, ${ }^{122}$ M. Malberti, ${ }^{122}$ H. Nguyen, ${ }^{122}$ A. Shrinivas, ${ }^{122}$ J. Sturdy, ${ }^{122}$ S. Sumowidagdo, ${ }^{122}$ S. Wimpenny, ${ }^{122}$ W. Andrews, ${ }^{123}$ J. G. Branson, ${ }^{123}$ G. B. Cerati, ${ }^{123}$ S. Cittolin, ${ }^{123}$ R. T. D'Agnolo, ${ }^{123}$ D. Evans, ${ }^{123}$ A. Holzner, ${ }^{123}$ R. Kelley, ${ }^{123}$ D. Kovalskyi, ${ }^{123}$ M. Lebourgeois, ${ }^{123}$ J. Letts, ${ }^{123}$ I. Macneill, ${ }^{123}$ S. Padhi, ${ }^{123}$ C. Palmer, ${ }^{123}$ M. Pieri, ${ }^{123}$ M. Sani, ${ }^{123}$ V. Sharma, ${ }^{123}$ S. Simon, ${ }^{123}$ E. Sudano, ${ }^{123}$ M. Tadel,${ }^{123}$ Y. Tu ${ }^{123}$ A. Vartak, ${ }^{123}$ S. Wasserbaech, ${ }^{123 c c c}$ F. Würthwein, ${ }^{123}$ A. Yagil, ${ }^{123}$ J. Yoo, ${ }^{123}$ D. Barge, ${ }^{124}$ C. Campagnari, ${ }^{124}$ T. Danielson, ${ }^{124}$ K. Flowers, ${ }^{124}$ P. Geffert, ${ }^{124}$ C. George, ${ }^{124}$ F. Golf, ${ }^{124}$ J. Incandela, ${ }^{124}$ C. Justus, ${ }^{124}$ R. Magaña Villalba, ${ }^{124}$ N. Mccoll, ${ }^{124}$ V. Pavlunin, ${ }^{124}$ J. Richman, ${ }^{124}$ R. Rossin, ${ }^{124}$ D. Stuart, ${ }^{124}$ W. To, ${ }^{124}$ C. West, ${ }^{124}$ A. Apresyan, ${ }^{125}$ A. Bornheim, ${ }^{125}$ J. Bunn, ${ }^{125}$ Y. Chen, ${ }^{125}$ E. Di Marco, ${ }^{125}$ J. Duarte, ${ }^{125}$ D. Kcira ${ }^{125}$ A. Mott, ${ }^{125}$ H. B. Newman, ${ }^{125}$ C. Pena, ${ }^{125}$ C. Rogan, ${ }^{125}$ M. Spiropulu, ${ }^{125}$ V. Timciuc,${ }^{125}$ R. Wilkinson, ${ }^{125}$ S. Xie, ${ }^{125}$ R. Y. Zhu ${ }^{125}$ V. Azzolini, ${ }^{126}$ A. Calamba, ${ }^{126}$ R. Carroll,,${ }^{126}$ T. Ferguson, ${ }^{126}$ Y. Iiyama, ${ }^{126}$ D. W. Jang, ${ }^{126}$ M. Paulini, ${ }^{126}$ J. Russ, ${ }^{126}$ H. Vogel, ${ }^{126}$ I. Vorobiev, ${ }^{126}$ J. P. Cumalat, ${ }^{127}$ B. R. Drell, ${ }^{127}$ W. T. Ford, ${ }^{127}$ A. Gaz, ${ }^{127}$ E. Luiggi Lopez, ${ }^{127}$ U. Nauenberg, ${ }^{127}$ J. G. Smith, ${ }^{127}$ K. Stenson, ${ }^{127}$ K. A. Ulmer, ${ }^{127}$ S. R. Wagner, ${ }^{127}$ J. Alexander, ${ }^{128}$ A. Chatterjee, ${ }^{128}$ N. Eggert, ${ }^{128}$ L. K. Gibbons,${ }^{128}$ W. Hopkins, ${ }^{128}$ A. Khukhunaishvili, ${ }^{128}$ B. Kreis, ${ }^{128}$ N. Mirman, ${ }^{128}$ G. Nicolas Kaufman, ${ }^{128}$ J. R. Patterson, ${ }^{128}$ A. Ryd, ${ }^{128}$ E. Salvati, ${ }^{128}$ W. Sun, ${ }^{128}$ W. D. Teo, ${ }^{128}$ J. Thom, ${ }^{128}$ J. Thompson, ${ }^{128}$ J. Tucker, ${ }^{128}$ Y. Weng, ${ }^{128}$ L. Winstrom, ${ }^{128}$ P. Wittich, ${ }^{128}$ D. Winn, ${ }^{129}$ S. Abdullin, ${ }^{130}$ 
M. Albrow, ${ }^{130}$ J. Anderson, ${ }^{130}$ G. Apollinari, ${ }^{130}$ L. A. T. Bauerdick, ${ }^{130}$ A. Beretvas, ${ }^{130}$ J. Berryhill, ${ }^{130}$ P. C. Bhat, ${ }^{130}$ K. Burkett, ${ }^{130}$ J. N. Butler, ${ }^{130}$ V. Chetluru, ${ }^{130}$ H. W. K. Cheung, ${ }^{130}$ F. Chlebana, ${ }^{130}$ S. Cihangir, ${ }^{130}$ V. D. Elvira, ${ }^{130}$ I. Fisk, ${ }^{130}$ J. Freeman, ${ }^{130}$ Y. Gao, ${ }^{130}$ E. Gottschalk, ${ }^{130}$ L. Gray, ${ }^{130}$ D. Green, ${ }^{130}$ S. Grünendahl, ${ }^{130}$ O. Gutsche, ${ }^{130}$ D. Hare, ${ }^{130}$ R. M. Harris, ${ }^{130}$ J. Hirschauer, ${ }^{130}$ B. Hooberman, ${ }^{130}$ S. Jindariani, ${ }^{130}$ M. Johnson, ${ }^{130}$ U. Joshi, ${ }^{130}$ K. Kaadze,${ }^{130}$ B. Klima, ${ }^{130}$ S. Kwan, ${ }^{130}$ J. Linacre, ${ }^{130}$ D. Lincoln, ${ }^{130}$ R. Lipton, ${ }^{130}$ J. Lykken, ${ }^{130}$ K. Maeshima, ${ }^{130}$ J. M. Marraffino, ${ }^{130}$ V. I. Martinez Outschoorn, ${ }^{130}$ S. Maruyama, ${ }^{130}$ D. Mason, ${ }^{130}$ P. McBride ${ }^{130}$ K. Mishra, ${ }^{130}$ S. Mrenna, ${ }^{130}$ Y. Musienko, ${ }^{130 h h}$ S. Nahn, ${ }^{130}$

C. Newman-Holmes, ${ }^{130}$ V. O’Dell, ${ }^{130}$ O. Prokofyev,${ }^{130}$ N. Ratnikova, ${ }^{130}$ E. Sexton-Kennedy, ${ }^{130}$ S. Sharma, ${ }^{130}$ W. J. Spalding, ${ }^{130}$ L. Spiegel, ${ }^{130}$ L. Taylor,${ }^{130}$ S. Tkaczyk, ${ }^{130}$ N. V. Tran, ${ }^{130}$ L. Uplegger, ${ }^{130}$ E. W. Vaandering,,${ }^{130}$ R. Vidal,,${ }^{130}$ A. Whitbeck, ${ }^{130}$ J. Whitmore, ${ }^{130}$ W. Wu, ${ }^{130}$ F. Yang, ${ }^{130}$ J. C. Yun, ${ }^{130}$ D. Acosta, ${ }^{131}$ P. Avery, ${ }^{131}$ D. Bourilkov, ${ }^{131}$ T. Cheng, ${ }^{131}$ S. Das, ${ }^{131}$ M. De Gruttola, ${ }^{131}$ G. P. Di Giovanni, ${ }^{131}$ D. Dobur, ${ }^{131}$ R. D. Field, ${ }^{131}$ M. Fisher, ${ }^{131}$ Y. Fu, ${ }^{131}$ I. K. Furic, ${ }^{131}$ J. Hugon, ${ }^{131}$ B. Kim ${ }^{131}$ J. Konigsberg, ${ }^{131}$ A. Korytov, ${ }^{131}$ A. Kropivnitskaya, ${ }^{131}$ T. Kypreos, ${ }^{131}$ J. F. Low, ${ }^{131}$ K. Matchev, ${ }^{131}$ P. Milenovic, ${ }^{131 d d d}$ G. Mitselmakher, ${ }^{131}$ L. Muniz, ${ }^{131}$ A. Rinkevicius, ${ }^{131}$ L. Shchutska,${ }^{131}$ N. Skhirtladze,${ }^{131}$ M. Snowball, ${ }^{131}$ J. Yelton, ${ }^{131}$ M. Zakaria, ${ }^{131}$ V. Gaultney, ${ }^{132}$ S. Hewamanage, ${ }^{132}$ S. Linn, ${ }^{132}$ P. Markowitz, ${ }^{132}$ G. Martinez, ${ }^{132}$ J. L. Rodriguez, ${ }^{132}$ T. Adams, ${ }^{133}$ A. Askew, ${ }^{133}$ J. Bochenek, ${ }^{133}$ J. Chen, ${ }^{133}$ B. Diamond, ${ }^{133}$ J. Haas, ${ }^{133}$ S. Hagopian, ${ }^{133}$ V. Hagopian, ${ }^{133}$ K. F. Johnson, ${ }^{133}$ H. Prosper, ${ }^{133}$ V. Veeraraghavan, ${ }^{133}$ M. Weinberg, ${ }^{133}$ M. M. Baarmand, ${ }^{134}$ B. Dorney, ${ }^{134}$ M. Hohlmann, ${ }^{134}$ H. Kalakhety, ${ }^{134}$ F. Yumiceva, ${ }^{134}$ M. R. Adams, ${ }^{135}$ L. Apanasevich, ${ }^{135}$ V. E. Bazterra, ${ }^{135}$ R. R. Betts, ${ }^{135}$ I. Bucinskaite, ${ }^{135}$ R. Cavanaugh, ${ }^{135}$ O. Evdokimov, ${ }^{135}$ L. Gauthier, ${ }^{135}$ C. E. Gerber, ${ }^{135}$ D. J. Hofman, ${ }^{135}$ S. Khalatyan, ${ }^{135}$ P. Kurt, ${ }^{135}$ D. H. Moon, ${ }^{135}$ C. O'Brien, ${ }^{135}$ C. Silkworth, ${ }^{135}$ P. Turner, ${ }^{135}$ N. Varelas, ${ }^{135}$ U. Akgun, ${ }^{136}$ E. A. Albayrak, ${ }^{136 x x}$ B. Bilki, ${ }^{136 e e e}$ W. Clarida, ${ }^{136}$ K. Dilsiz, ${ }^{136}$ F. Duru, ${ }^{136}$ M. Haytmyradov, ${ }^{136}$ J.-P. Merlo, ${ }^{136}$ H. Mermerkaya, ${ }^{136 f f f}$ A. Mestvirishvili, ${ }^{136}$ A. Moeller, ${ }^{136}$ J. Nachtman, ${ }^{136}$ H. Ogul, ${ }^{136}$ Y. Onel, ${ }^{136}$ F. Ozok, ${ }^{136 x x}$ S. Sen, ${ }^{136}$ P. Tan, ${ }^{136}$ E. Tiras, ${ }^{136}$ J. Wetzel, ${ }^{136}$ T. Yetkin, ${ }^{136 g g}$ K. Yi, ${ }^{136}$ B. A. Barnett, ${ }^{137}$ B. Blumenfeld, ${ }^{137}$ S. Bolognesi, ${ }^{137}$ D. Fehling, ${ }^{137}$ A. V. Gritsan, ${ }^{137}$ P. Maksimovic, ${ }^{137}$ C. Martin, ${ }^{137}$ M. Swartz, ${ }^{137}$ P. Baringer, ${ }^{138}$ A. Bean, ${ }^{138}$ G. Benelli, ${ }^{138}$ R. P. Kenny III,${ }^{138}$ M. Murray, ${ }^{138}$ D. Noonan, ${ }^{138}$ S. Sanders, ${ }^{138}$ J. Sekaric, ${ }^{138}$ R. Stringer, ${ }^{138}$ Q. Wang, ${ }^{138}$ J. S. Wood, ${ }^{138}$ A. F. Barfuss, ${ }^{139}$ I. Chakaberia, ${ }^{139}$ A. Ivanov, ${ }^{139}$ S. Khalil, ${ }^{139}$ M. Makouski, ${ }^{139}$ Y. Maravin, ${ }^{139}$ L. K. Saini, ${ }^{139}$ S. Shrestha ${ }^{139}$ I. Svintradze,${ }^{139}$ J. Gronberg, ${ }^{140}$ D. Lange, ${ }^{140}$ F. Rebassoo, ${ }^{140}$ D. Wright, ${ }^{140}$ A. Baden, ${ }^{141}$ B. Calvert, ${ }^{141}$ S. C. Eno, ${ }^{141}$ J. A. Gomez, ${ }^{141}$ N. J. Hadley, ${ }^{141}$ R. G. Kellogg, ${ }_{141}$ T. Kolberg, ${ }^{141}$ Y. Lu, ${ }^{141}$ M. Marionneau, ${ }^{141}$ A. C. Mignerey, ${ }^{141}$ K. Pedro, ${ }^{141}$ A. Skuja, ${ }^{141}$ J. Temple, ${ }^{141}$ M. B. Tonjes, ${ }^{141}$ S. C. Tonwar, ${ }^{141}$ A. Apyan, ${ }^{142}$ R. Barbieri, ${ }^{142}$ G. Bauer, ${ }^{142}$ W. Busza, ${ }^{142}$ I. A. Cali, ${ }^{142}$ M. Chan, ${ }^{142}$ L. Di Matteo, ${ }^{142}$ V. Dutta, ${ }^{142}$ G. Gomez Ceballos, ${ }^{142}$ M. Goncharov, ${ }^{142}$ D. Gulhan, ${ }^{142}$ M. Klute, ${ }^{142}$ Y. S. Lai, ${ }^{142}$ Y.-J. Lee,${ }^{142}$ A. Levin, ${ }^{142}$ P. D. Luckey, ${ }^{142}$ T. Ma ${ }^{142}$ C. Paus,${ }^{142}$ D. Ralph, ${ }^{142}$ C. Roland, ${ }^{142}$ G. Roland, ${ }^{142}$ G. S. F. Stephans, ${ }^{142}$ F. Stöckli, ${ }^{142}$ K. Sumorok, ${ }^{142}$ D. Velicanu, ${ }^{142}$ J. Veverka, ${ }^{142}$ B. Wyslouch,,${ }^{142}$ M. Yang, ${ }^{142}$ A. S. Yoon, ${ }^{142}$ M. Zanetti, ${ }^{142}$ V. Zhukova, ${ }^{142}$ B. Dahmes, ${ }^{143}$ A. De Benedetti, ${ }^{143}$ A. Gude, ${ }^{143}$ S. C. Kao,${ }^{143}$ K. Klapoetke, ${ }^{143}$ Y. Kubota,${ }^{143}$ J. Mans, ${ }^{143}$ N. Pastika, ${ }^{143}$ R. Rusack, ${ }^{143}$ A. Singovsky, ${ }^{143}$ N. Tambe, ${ }^{143}$ J. Turkewitz, ${ }^{143}$ J. G. Acosta, ${ }^{144}$ L. M. Cremaldi, ${ }^{144}$

R. Kroeger, ${ }^{144}$ S. Oliveros, ${ }^{144}$ L. Perera,${ }^{144}$ R. Rahmat, ${ }^{144}$ D. A. Sanders, ${ }^{144}$ D. Summers, ${ }^{144}$ E. Avdeeva, ${ }^{145}$ K. Bloom, ${ }^{145}$ S. Bose,${ }^{145}$ D. R. Claes, ${ }^{145}$ A. Dominguez, ${ }^{145}$ R. Gonzalez Suarez, ${ }^{145}$ J. Keller, ${ }^{145}$ D. Knowlton, ${ }^{145}$ I. Kravchenko, ${ }^{145}$ J. LazoFlores, ${ }^{145}$ S. Malik, ${ }^{145}$ F. Meier, ${ }^{145}$ G. R. Snow, ${ }^{145}$ J. Dolen, ${ }^{146}$ A. Godshalk, ${ }^{146}$ I. Iashvili, ${ }^{146}$ S. Jain, ${ }^{146}$ A. Kharchilava, ${ }^{146}$ A. Kumar, ${ }^{146}$ S. Rappoccio, ${ }^{146}$ G. Alverson, ${ }^{147}$ E. Barberis,${ }^{147}$ D. Baumgartel,,${ }^{147}$ M. Chasco, ${ }^{147}$ J. Haley, ${ }^{147}$ A. Massironi, ${ }^{147}$ D. Nash, ${ }^{147}$ T. Orimoto, ${ }^{147}$ D. Trocino, ${ }^{147}$ D. Wood, ${ }^{147}$ J. Zhang, ${ }^{147}$ A. Anastassov, ${ }^{148}$ K. A. Hahn, ${ }^{148}$ A. Kubik, ${ }^{148}$ L. Lusito, ${ }_{148}^{148}$ N. Mucia, ${ }^{148}$ N. Odell, ${ }^{148}$ B. Pollack, ${ }^{148}$ A. Pozdnyakov, ${ }^{148}$ M. Schmitt, ${ }^{148}$ S. Stoynev, ${ }^{148}$ K. Sung, ${ }^{148}$ M. Velasco, ${ }^{148}$ S. Won, ${ }^{148}$ D. Berry, ${ }^{149}$ A. Brinkerhoff, ${ }^{149}$ K. M. Chan, ${ }^{149}$ A. Drozdetskiy, ${ }^{149}$ M. Hildreth,${ }^{149}$ C. Jessop, ${ }^{149}$ D. J. Karmgard ${ }^{149}$ N. Kellams, ${ }^{149}$ J. Kolb, ${ }^{149}$ K. Lannon, ${ }^{149}$ W. Luo, ${ }^{149}$ S. Lynch, ${ }^{149}$ N. Marinelli, ${ }^{149}$ D. M. Morse, ${ }^{149}$ T. Pearson, ${ }^{149}$ M. Planer, ${ }^{149}$ R. Ruchti, ${ }^{149}$ J. Slaunwhite, ${ }^{149}$ N. Valls, ${ }^{149}$ M. Wayne, ${ }^{149}$ M. Wolf, ${ }^{149}$ A. Woodard, ${ }^{149}$ L. Antonelli, ${ }^{150}$ B. Bylsma, ${ }^{150}$ L. S. Durkin, ${ }^{150}$ S. Flowers, ${ }^{150}$ C. Hill,${ }^{150}$ R. Hughes, ${ }^{150}$ K. Kotov, ${ }^{150}$ T. Y. Ling, ${ }^{150}$ D. Puigh, ${ }^{150}$ M. Rodenburg, ${ }^{150}$ G. Smith ${ }^{150}$ C. Vuosalo, ${ }^{150}$ B. L. Winer ${ }^{150}$ H. Wolfe,${ }^{150}$ H. W. Wulsin, ${ }^{150}$ E. Berry, ${ }^{151}$ P. Elmer, ${ }^{151}$ V. Halyo, ${ }^{151}$ P. Hebda,${ }^{151}$ J. Hegeman, ${ }^{151}$ A. Hunt, ${ }^{151}$ P. Jindal,${ }^{151}$ S. A. Koay, ${ }^{151}$ P. Lujan, ${ }^{151}$ D. Marlow, ${ }^{151}$ T. Medvedeva, ${ }^{151}$ M. Mooney, ${ }_{151}$ J. Olsen, ${ }^{151}$ P. Piroué, ${ }^{151}$ X. Quan, ${ }^{151}$ A. Raval ${ }^{151}$ H. Saka, ${ }^{151}$ D. Stickland, ${ }^{151}$ C. Tully, ${ }^{151}$ J. S. Werner, ${ }^{151}$ S. C. Zenz, ${ }^{151}$ A. Zuranski, ${ }^{151}$ E. Brownson, ${ }^{152}$ A. Lopez, ${ }^{152}$ H. Mendez, ${ }^{152}$ J. E. Ramirez Vargas, ${ }^{152}$ E. Alagoz,${ }^{153}$ D. Benedetti, ${ }^{153}$ G. Bolla, ${ }^{153}$ D. Bortoletto, ${ }^{153}$ M. De Mattia, ${ }^{153}$ A. Everett, ${ }^{153}$ Z. Hu, ${ }^{153}$ M. Jha, ${ }^{153}$ M. Jones,${ }^{153}$ K. Jung, ${ }^{153}$ M. Kress,${ }^{153}$ N. Leonardo, ${ }^{153}$ D. Lopes Pegna, ${ }^{153}$ V. Maroussov,${ }^{153}$ P. Merkel, ${ }^{153}$ D. H. Miller, ${ }^{153}$ N. Neumeister, ${ }^{153}$ B. C. Radburn-Smith, ${ }^{153}$ I. Shipsey, ${ }^{153}$ D. Silvers, ${ }^{153}$ A. Svyatkovskiy, ${ }^{153}$ F. Wang, ${ }^{153}$ W. Xie, ${ }^{153}$ L. Xu, ${ }^{153}$ 
H. D. Yoo, ${ }^{153}$ J. Zablocki, ${ }^{153}$ Y. Zheng, ${ }^{153}$ N. Parashar, ${ }^{154}$ A. Adair, ${ }^{155}$ B. Akgun, ${ }^{155}$ K. M. Ecklund, ${ }^{155}$ F. J. M. Geurts, ${ }^{155}$ W. Li ${ }^{155}$ B. Michlin, ${ }^{155}$ B. P. Padley, ${ }^{155}$ R. Redjimi, ${ }^{155}$ J. Roberts, ${ }^{155}$ J. Zabel, ${ }^{155}$ B. Betchart, ${ }^{156}$ A. Bodek, ${ }^{156}$ R. Covarelli, ${ }^{156}$ P. de Barbaro, ${ }^{156}$ R. Demina, ${ }^{156}$ Y. Eshaq, ${ }^{156}$ T. Ferbel, ${ }^{156}$ A. Garcia-Bellido, ${ }^{156}$ P. Goldenzweig,,${ }^{156}$ J. Han, ${ }^{156}$ A. Harel, ${ }^{156}$ D. C. Miner, ${ }^{156}$ G. Petrillo, ${ }^{156}$ D. Vishnevskiy, ${ }^{156}$ M. Zielinski, ${ }^{156}$ A. Bhatti, ${ }^{157}$ R. Ciesielski, ${ }^{157}$ L. Demortier, ${ }^{157}$ K. Goulianos, ${ }^{157}$ G. Lungu, ${ }^{157}$ S. Malik, ${ }^{157}$ C. Mesropian, ${ }^{157}$ S. Arora, ${ }^{158}$ A. Barker ${ }^{158}$ J. P. Chou, ${ }^{158}$ C. ContrerasCampana, ${ }^{158}$ E. Contreras-Campana, ${ }^{158}$ D. Duggan, ${ }^{158}$ D. Ferencek, ${ }^{158}$ Y. Gershtein, ${ }^{158}$ R. Gray, ${ }^{158}$ E. Halkiadakis, ${ }^{158}$ D. Hidas, ${ }^{158}$ A. Lath,${ }^{158}$ S. Panwalkar, ${ }^{158}$ M. Park, ${ }^{158}$ R. Patel, ${ }^{158}$ V. Rekovic, ${ }^{158}$ J. Robles, ${ }^{158}$ S. Salur, ${ }^{158}$ S. Schnetzer, ${ }^{158}$ C. Seitz, ${ }^{158}$ S. Somalwar, ${ }^{158}$ R. Stone ${ }^{158}$ S. Thomas, ${ }^{158}$ P. Thomassen, ${ }^{158}$ M. Walker, ${ }^{158}$ K. Rose, ${ }^{159}$ S. Spanier, ${ }^{159}$ Z. C. Yang, ${ }^{159}$ A. York, ${ }^{159}$ O. Bouhali, ${ }^{160 h h}$ R. Eusebi, ${ }^{160}$ W. Flanagan, ${ }^{160}$ J. Gilmore, ${ }^{160}$ T. Kamon, ${ }^{160 i i}$ V. Khotilovich, ${ }^{160}$ V. Krutelyov, ${ }^{160}$ R. Montalvo, ${ }^{160}$ I. Osipenkov, ${ }^{160}$ Y. Pakhotin, ${ }^{160}$ A. Perloff, ${ }^{160}$ J. Roe, ${ }^{160}$ A. Safonov, ${ }^{160}$ T. Sakuma, ${ }^{160}$ I. Suarez, ${ }^{160}$ A. Tatarinov, ${ }^{160}$ D. Toback,${ }^{160}$ N. Akchurin, ${ }^{161}$ C. Cowden,${ }^{161}$ J. Damgov, ${ }^{161}$ C. Dragoiu, ${ }^{161}$ P. R. Dudero, ${ }^{161}$

K. Kovitanggoon, ${ }^{161}$ S. Kunori, ${ }^{161}$ S. W. Lee, ${ }^{161}$ T. Libeiro, ${ }^{161}$ I. Volobouev, ${ }^{161}$ E. Appelt, ${ }^{162}$ A. G. Delannoy, ${ }^{162}$ S. Greene, ${ }^{162}$ A. Gurrola, ${ }^{162}$ W. Johns, ${ }^{162}$ C. Maguire, ${ }^{162}$ Y. Mao, ${ }^{162}$ A. Melo, ${ }^{162}$ M. Sharma, ${ }^{162}$ P. Sheldon, ${ }^{162}$ B. Snook, ${ }^{162}$ S. Tuo, ${ }^{162}$ J. Velkovska, ${ }^{162}$ M. W. Arenton, ${ }^{163}$ S. Boutle, ${ }^{163}$ B. Cox,${ }^{163}$ B. Francis, ${ }^{163}$ J. Goodell, ${ }^{163}$ R. Hirosky, ${ }^{163}$ A. Ledovskoy, ${ }^{163}$ C. Lin, ${ }^{163}$ C. Neu, ${ }^{163}$ J. Wood,${ }^{163}$ S. Gollapinni, ${ }^{164}$ R. Harr,${ }^{164}$ P. E. Karchin, ${ }^{164}$ C. Kottachchi Kankanamge Don, ${ }^{164}$ P. Lamichhane, ${ }^{164}$ D. A. Belknap, ${ }^{165}$ L. Borrello, ${ }^{165}$ D. Carlsmith,${ }^{165}$ M. Cepeda, ${ }^{165}$ S. Dasu, ${ }^{165}$ S. Duric, ${ }^{165}$ E. Friis, ${ }^{165}$ M. Grothe, ${ }^{165}$ R. Hall-Wilton, ${ }^{165}$ M. Herndon, ${ }^{165}$ A. Hervé, ${ }^{165}$ P. Klabbers, ${ }^{165}$ J. Klukas, ${ }^{165}$ A. Lanaro, ${ }^{165}$ A. Levine, ${ }^{165}$ R. Loveless, ${ }^{165}$ A. Mohapatra ${ }^{165}$ I. Ojalvo, ${ }^{165}$ T. Perry,${ }^{165}$ G. A. Pierro, ${ }^{165}$ G. Polese,${ }^{165}$ I. Ross, ${ }^{165}$ A. Sakharov, ${ }^{165}$ T. Sarangi, ${ }^{165}$ A. Savin ${ }^{165}$ and W. H. Smith ${ }^{165}$

(CMS Collaboration)

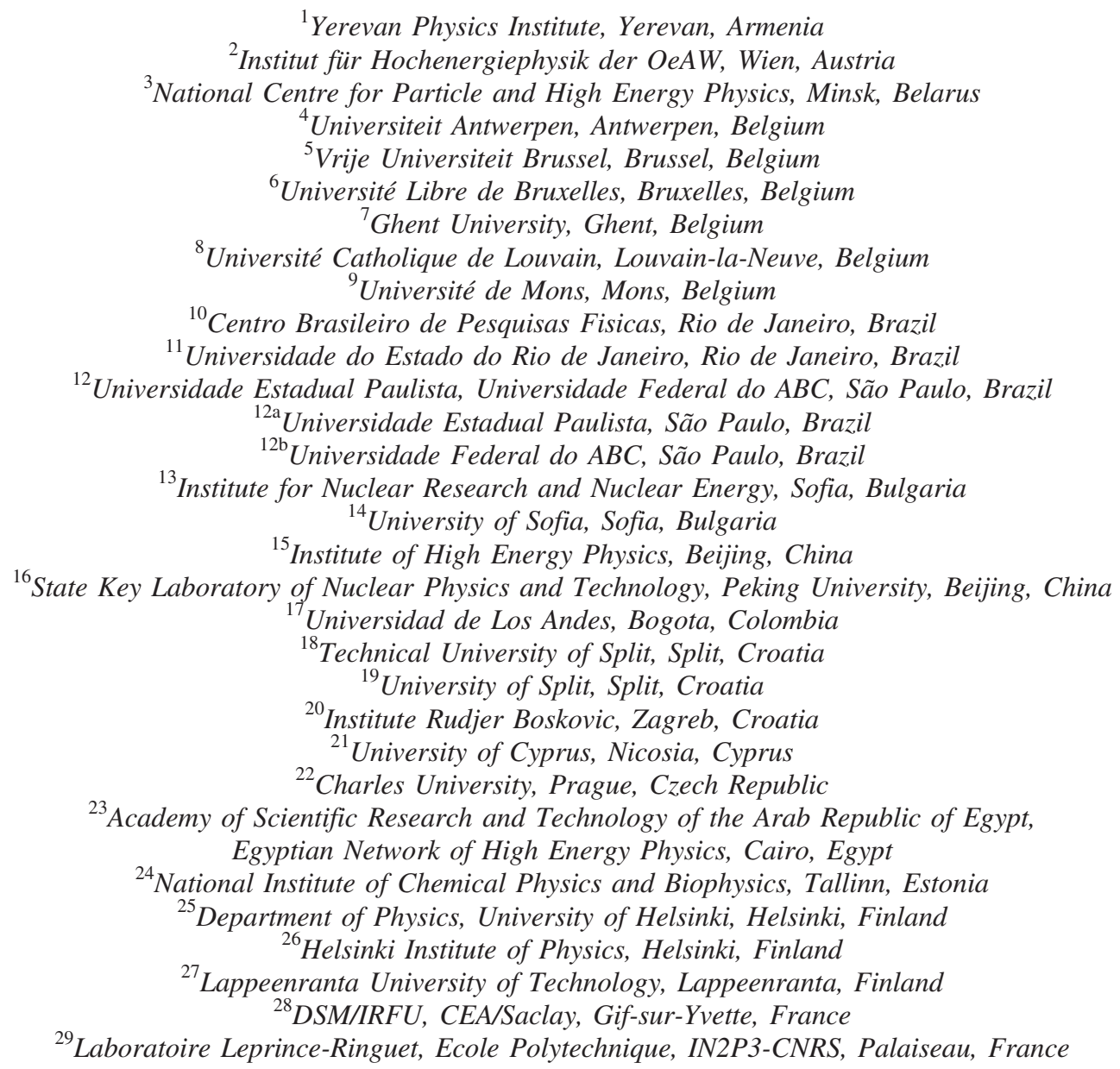


${ }^{30}$ Institut Pluridisciplinaire Hubert Curien, Université de Strasbourg,

Université de Haute Alsace Mulhouse, CNRS/IN2P3, Strasbourg, France

${ }^{31}$ Centre de Calcul de l'Institut National de Physique Nucleaire et de Physique des Particules,

CNRS/IN2P3, Villeurbanne, France

${ }^{32}$ Université de Lyon, Université Claude Bernard Lyon 1, CNRS-IN2P3,

Institut de Physique Nucléaire de Lyon, Villeurbanne, France

${ }^{33}$ Institute of High Energy Physics and Informatization, Tbilisi State University, Tbilisi, Georgia

${ }^{34}$ RWTH Aachen University, I. Physikalisches Institut, Aachen, Germany

${ }^{35}$ RWTH Aachen University, III. Physikalisches Institut A, Aachen, Germany

${ }^{36}$ RWTH Aachen University, III. Physikalisches Institut B, Aachen, Germany

${ }^{37}$ Deutsches Elektronen-Synchrotron, Hamburg, Germany

${ }^{38}$ University of Hamburg, Hamburg, Germany

${ }^{39}$ Institut für Experimentelle Kernphysik, Karlsruhe, Germany

${ }^{40}$ Institute of Nuclear and Particle Physics (INPP), NCSR Demokritos, Aghia Paraskevi, Greece

${ }^{41}$ University of Athens, Athens, Greece

${ }^{42}$ University of Ioánnina, Ioánnina, Greece

${ }^{43}$ Wigner Research Centre for Physics, Budapest, Hungary

${ }^{44}$ Institute of Nuclear Research ATOMKI, Debrecen, Hungary

${ }^{45}$ University of Debrecen, Debrecen, Hungary

${ }^{46}$ National Institute of Science Education and Research, Bhubaneswar, India

${ }^{47}$ Panjab University, Chandigarh, India

${ }^{48}$ University of Delhi, Delhi, India

${ }^{49}$ Saha Institute of Nuclear Physics, Kolkata, India

${ }^{50}$ Bhabha Atomic Research Centre, Mumbai, India

${ }^{51}$ Tata Institute of Fundamental Research - EHEP, Mumbai, India

${ }^{52}$ Tata Institute of Fundamental Research - HECR, Mumbai, India

${ }^{53}$ Institute for Research in Fundamental Sciences (IPM), Tehran, Iran

${ }^{54}$ University College Dublin, Dublin, Ireland

${ }^{55 \mathrm{a} I N F N}$ Sezione di Bari, Bari, Italy

${ }^{55 \mathrm{~b}}$ Università di Bari, Bari, Italy

${ }^{55 \mathrm{c}}$ Politecnico di Bari, Bari, Italy

${ }^{56 \mathrm{a} I N F N}$ Sezione di Bologna, Bologna, Italy

${ }^{56 \mathrm{~b}}$ Università di Bologna, Bologna, Italy

${ }^{57 a}$ INFN Sezione di Catania, Catania, Italy

${ }^{57 b}$ Università di Catania, Catania, Italy

${ }^{57 \mathrm{C}}$ CSFNSM, Catania, Italy

${ }^{58 \mathrm{a}}$ INFN Sezione di Firenze, Firenze, Italy

${ }^{58 \mathrm{~b}}$ Università di Firenze, Firenze, Italy

${ }^{59}$ INFN Laboratori Nazionali di Frascati, Frascati, Italy

${ }^{60 a}$ INFN Sezione di Genova, Genova, Italy

${ }^{60 \mathrm{~b}}$ Università di Genova, Genova, Italy

${ }^{61 a}$ INFN Sezione di Milano-Bicocca, Milano, Italy

${ }^{61 \mathrm{~b}}$ Università di Milano-Bicocca, Milano, Italy

${ }^{62 \mathrm{a}}$ INFN Sezione di Napoli, Napoli, Italy

${ }^{62 \mathrm{~b}}$ Università di Napoli 'Federico II', Napoli, Italy

${ }^{62 \mathrm{c}}$ Università della Basilicata (Potenza), Napoli, Italy

${ }^{62 \mathrm{~d}}$ Università G. Marconi (Roma), Napoli, Italy

${ }^{63 a}$ INFN Sezione di Padova, Padova, Italy

${ }^{63 \mathrm{~b}}$ Università di Padova, Padova, Italy

${ }^{63 c}$ Università di Trento (Trento), Padova, Italy

${ }^{64 a}$ INFN Sezione di Pavia, Pavia, Italy

${ }^{64 \mathrm{~b}}$ Università di Pavia, Pavia, Italy

${ }^{65}$ INFN Sezione di Perugia, Perugia, Italy

${ }^{65 \mathrm{~b}}$ Università di Perugia, Perugia, Italy

${ }^{66 a}$ INFN Sezione di Pisa, Pisa, Italy

${ }^{66 \mathrm{~b}}$ Università di Pisa, Pisa, Italy

${ }^{66 c}$ Scuola Normale Superiore di Pisa, Pisa, Italy

${ }^{67 a}$ INFN Sezione di Roma, Roma, Italy

${ }^{67 \mathrm{~b}}$ Università di Roma, Roma, Italy

${ }^{68 \mathrm{a}}$ INFN Sezione di Torino, Torino, Italy 
${ }^{68 \mathrm{~b}}$ Università di Torino, Torino, Italy

${ }^{68 \mathrm{c}}$ Università del Piemonte Orientale (Novara), Torino, Italy

${ }^{69}$ INFN Sezione di Trieste, Trieste, Italy

${ }^{69 \mathrm{~b}}$ Università di Trieste, Trieste, Italy

${ }^{70}$ Kangwon National University, Chunchon, Korea

${ }^{71}$ Kyungpook National University, Daegu, Korea

${ }^{72}$ Chonnam National University, Institute for Universe and Elementary Particles, Kwangju, Korea

${ }^{73}$ Korea University, Seoul, Korea

${ }^{74}$ University of Seoul, Seoul, Korea

${ }^{75}$ Sungkyunkwan University, Suwon, Korea

${ }^{76}$ Vilnius University, Vilnius, Lithuania

${ }^{77}$ University of Malaya Jabatan Fizik, Kuala Lumpur, Malaysia

${ }^{78}$ Centro de Investigacion y de Estudios Avanzados del IPN, Mexico City, Mexico

${ }^{79}$ Universidad Iberoamericana, Mexico City, Mexico

${ }^{80}$ Benemerita Universidad Autonoma de Puebla, Puebla, Mexico

${ }^{81}$ Universidad Autónoma de San Luis Potosí, San Luis Potosí, Mexico

${ }^{82}$ University of Auckland, Auckland, New Zealand

${ }^{83}$ University of Canterbury, Christchurch, New Zealand

${ }^{84}$ National Centre for Physics, Quaid-I-Azam University, Islamabad, Pakistan

${ }^{85}$ National Centre for Nuclear Research, Swierk, Poland

${ }^{86}$ Institute of Experimental Physics, Faculty of Physics, University of Warsaw, Warsaw, Poland

${ }^{87}$ Laboratório de Instrumentação e Física Experimental de Partículas, Lisboa, Portugal

${ }^{88}$ Joint Institute for Nuclear Research, Dubna, Russia

${ }^{89}$ Petersburg Nuclear Physics Institute, Gatchina (St. Petersburg), Russia

${ }^{90}$ Institute for Nuclear Research, Moscow, Russia

${ }^{91}$ Institute for Theoretical and Experimental Physics, Moscow, Russia

${ }_{92}$ P.N. Lebedev Physical Institute, Moscow, Russia

${ }^{93}$ Skobeltsyn Institute of Nuclear Physics, Lomonosov Moscow State University, Moscow, Russia

${ }^{94}$ State Research Center of Russian Federation, Institute for High Energy Physics, Protvino, Russia

${ }^{95}$ University of Belgrade, Faculty of Physics and Vinca Institute of Nuclear Sciences, Belgrade, Serbia

${ }^{96}$ Centro de Investigaciones Energéticas Medioambientales y Tecnológicas (CIEMAT), Madrid, Spain

${ }^{97}$ Universidad Autónoma de Madrid, Madrid, Spain

${ }^{98}$ Universidad de Oviedo, Oviedo, Spain

${ }^{99}$ Instituto de Física de Cantabria (IFCA), CSIC-Universidad de Cantabria, Santander, Spain

${ }^{100}$ CERN, European Organization for Nuclear Research, Geneva, Switzerland

${ }^{101}$ Paul Scherrer Institut, Villigen, Switzerland

${ }^{102}$ Institute for Particle Physics, ETH Zurich, Zurich, Switzerland

${ }^{103}$ Universität Zürich, Zurich, Switzerland

${ }^{104}$ National Central University, Chung-Li, Taiwan

${ }^{105}$ National Taiwan University (NTU), Taipei, Taiwan

${ }^{106}$ Chulalongkorn University, Bangkok, Thailand

${ }^{107}$ Cukurova University, Adana, Turkey

${ }^{108}$ Middle East Technical University, Physics Department, Ankara, Turkey

${ }^{109}$ Bogazici University, Istanbul, Turkey

${ }^{110}$ Istanbul Technical University, Istanbul, Turkey

${ }^{111}$ National Scientific Center, Kharkov Institute of Physics and Technology, Kharkov, Ukraine

${ }^{112}$ University of Bristol, Bristol, United Kingdom

${ }^{113}$ Rutherford Appleton Laboratory, Didcot, United Kingdom

${ }^{114}$ Imperial College, London, United Kingdom

${ }^{115}$ Brunel University, Uxbridge, United Kingdom

${ }^{116}$ Baylor University, Waco, USA

${ }^{117}$ The University of Alabama, Tuscaloosa, USA

${ }^{118}$ Boston University, Boston, USA

${ }^{119}$ Brown University, Providence, USA

${ }^{120}$ University of California, Davis, Davis, USA

${ }^{121}$ University of California, Los Angeles, USA

${ }^{122}$ University of California, Riverside, Riverside, USA

${ }^{123}$ University of California, San Diego, La Jolla, USA

${ }^{124}$ University of California, Santa Barbara, Santa Barbara, USA

${ }^{125}$ California Institute of Technology, Pasadena, USA 


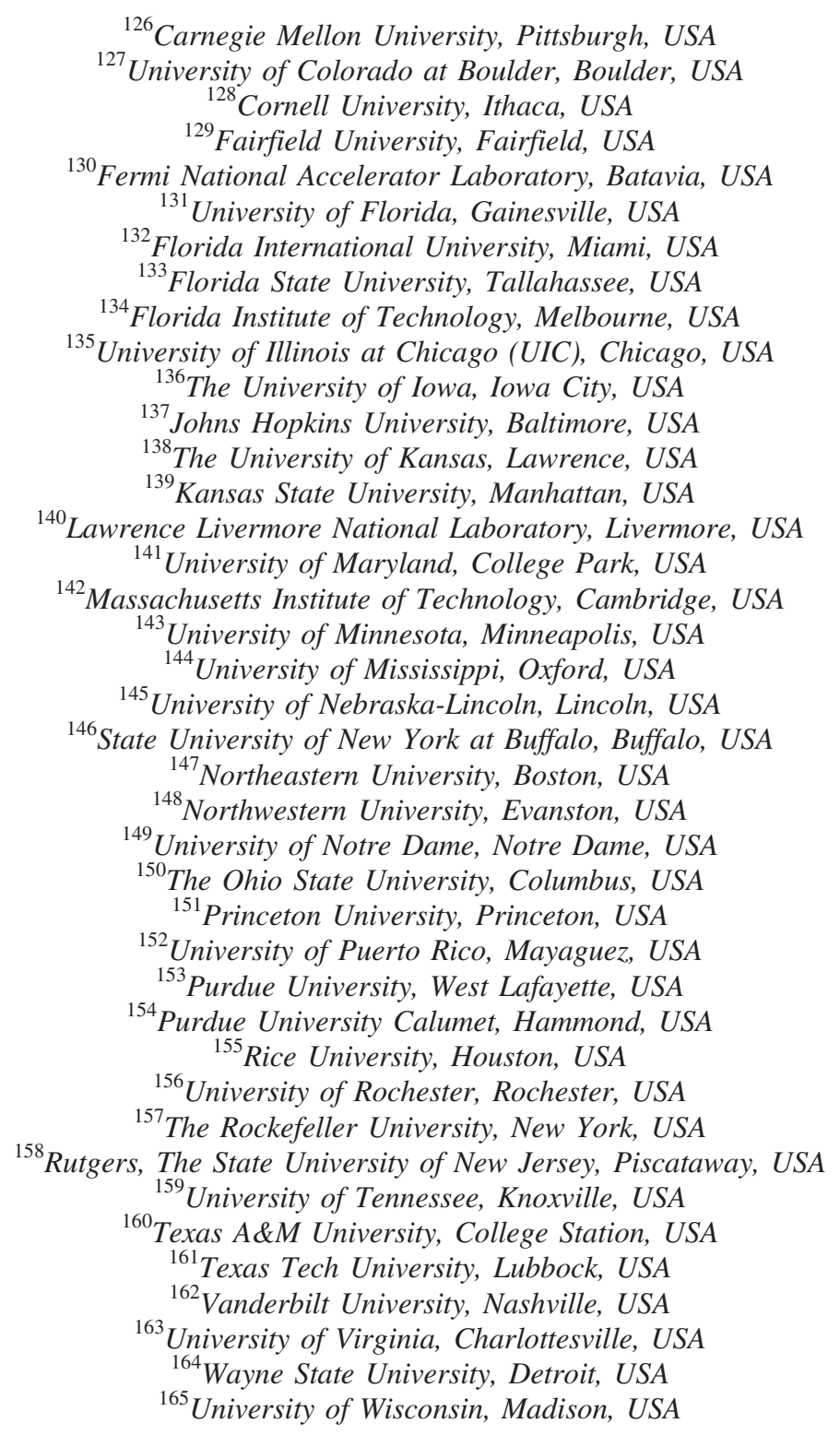

${ }^{\mathrm{a}}$ Deceased.

${ }^{\mathrm{b}}$ Also at Vienna University of Technology, Vienna, Austria.

${ }^{\mathrm{c}}$ Also at CERN, European Organization for Nuclear Research, Geneva, Switzerland.

${ }^{\mathrm{d}}$ Also at Institut Pluridisciplinaire Hubert Curien, Université de Strasbourg, Université de Haute Alsace Mulhouse, CNRS/IN2P3, Strasbourg, France.

${ }^{\mathrm{e}}$ Also at National Institute of Chemical Physics and Biophysics, Tallinn, Estonia.

${ }^{\mathrm{f}}$ Also at Skobeltsyn Institute of Nuclear Physics, Lomonosov Moscow State University, Moscow, Russia.

${ }^{\mathrm{g}}$ Also at Universidade Estadual de Campinas, Campinas, Brazil.

${ }^{\mathrm{h}}$ Also at California Institute of Technology, Pasadena, USA.

${ }^{\mathrm{i}}$ Also at Laboratoire Leprince-Ringuet, Ecole Polytechnique, IN2P3-CNRS, Palaiseau, France.

${ }^{\mathrm{j}}$ Also at Zewail City of Science and Technology, Zewail, Egypt.

${ }^{\mathrm{k}}$ Also at Suez Canal University, Suez, Egypt.

${ }^{1}$ Also at Cairo University, Cairo, Egypt.

${ }^{\mathrm{m}}$ Also at Fayoum University, El-Fayoum, Egypt.

${ }^{\mathrm{n}}$ Also at British University in Egypt, Cairo, Egypt.

${ }^{\circ}$ Present address: Ain Shams University, Cairo, Egypt.

${ }^{\mathrm{p}}$ Also at Université de Haute Alsace, Mulhouse, France.

${ }^{\mathrm{q}}$ Also at Joint Institute for Nuclear Research, Dubna, Russia.

${ }^{\mathrm{r}}$ Also at Brandenburg University of Technology, Cottbus, Germany. 
${ }^{\mathrm{s}}$ Also at The University of Kansas, Lawrence, USA.

${ }^{t}$ Also at Institute of Nuclear Research ATOMKI, Debrecen, Hungary.

${ }^{u}$ Also at Eötvös Loránd University, Budapest, Hungary.

${ }^{v}$ Also at Tata Institute of Fundamental Research - HECR, Mumbai, India.

${ }^{w}$ Present address: King Abdulaziz University, Jeddah, Saudi Arabia.

${ }^{\mathrm{x}}$ Also at University of Visva-Bharati, Santiniketan, India.

${ }^{y}$ Also at University of Ruhuna, Matara, Sri Lanka.

${ }^{\mathrm{z}}$ Also at Isfahan University of Technology, Isfahan, Iran.

${ }^{\text {aa }}$ Also at Sharif University of Technology, Tehran, Iran.

${ }^{\mathrm{bb}}$ Also at Plasma Physics Research Center, Science and Research Branch, Islamic Azad University, Tehran, Iran.

${ }^{c c}$ Also at Università degli Studi di Siena, Siena, Italy.

${ }^{\mathrm{dd}}$ Also at Centre National de la Recherche Scientifique (CNRS) - IN2P3, Paris, France.

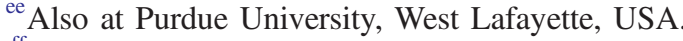

${ }^{\mathrm{ff}}$ Also at Universidad Michoacana de San Nicolas de Hidalgo, Morelia, Mexico.

${ }^{g g}$ Also at National Centre for Nuclear Research, Swierk, Poland.

${ }^{\text {hh }}$ Also at Institute for Nuclear Research, Moscow, Russia.

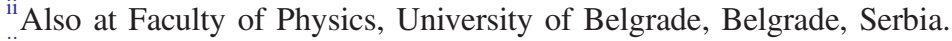

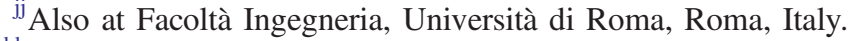

${ }^{\mathrm{kk}}$ Also at Scuola Normale e Sezione dell'INFN, Pisa, Italy.

${ }^{11}$ Also at University of Athens, Athens, Greece.

${ }^{\mathrm{mm}}$ Also at Paul Scherrer Institut, Villigen, Switzerland.

${ }^{n n}$ Also at Institute for Theoretical and Experimental Physics, Moscow, Russia.

${ }^{\mathrm{oo}}$ Also at Albert Einstein Center for Fundamental Physics, Bern, Switzerland.

${ }^{\mathrm{pp}}$ Also at Gaziosmanpasa University, Tokat, Turkey.

${ }^{\mathrm{qq}}$ Also at Adiyaman University, Adiyaman, Turkey.

${ }^{\mathrm{rr}}$ Also at Cag University, Mersin, Turkey.

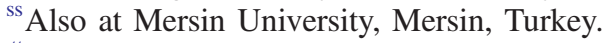

${ }^{t t}$ Also at Izmir Institute of Technology, Izmir, Turkey.

${ }^{u u}$ Also at Ozyegin University, Istanbul, Turkey.

${ }^{\mathrm{vv}}$ Also at Kafkas University, Kars, Turkey.

${ }^{\text {ww }}$ Also at Istanbul University, Faculty of Science, Istanbul, Turkey. 